\title{
Tailoring the separation performance of ZIF-based mixed matrix membranes by MOF-matrix interfacial compatibilization
}

\author{
Machiel van Essen ${ }^{\mathrm{a}}$, Raymond Thür ${ }^{\mathrm{b}}$, Luuk van den Akker ${ }^{\mathrm{a}}$, Menno Houben ${ }^{\mathrm{a}}$, Ivo F. \\ J. Vankelecom ${ }^{\mathrm{b}}$, Kitty Nijmeijer ${ }^{\mathrm{a}}$, Zandrie Borneman ${ }^{\mathrm{a},{ }^{*}}$ \\ ${ }^{a}$ Membrane Materials and Processes, Department of Chemical Engineering and Chemistry, Eindhoven University of Technology, P.0. Box 513, 5600 MB Eindhoven, the \\ Netherlands \\ bembrane Technology Group, CMACs, KU Leuven, Celestijnenlaan 200F, Box 2454, 3001 Heverlee, Belgium
}

\section{A R T I C L E I N F}

Keywords:

Mixed matrix membranes

Zeolitic imidazolate frameworks

Interfacial compatibilization

MOF-Matrix interface

$\mathrm{CO}_{2}$-Based separations

\begin{abstract}
A B S T R A C T
Controlling the metal-organic framework (MOF)-natrix interface is a useful strategy to improve the gas separation performance of mixed matrix membranes (MMMs). Although polymer blending has been investigated to enhance MMM performances, its true strength, i.e., aligning polymer and additive chemistries to improve interfacial compatibility and ultimately the separation performance, is only poorly investigated. In this work we demonstrate how controlling interfacial chemistries by polymer blending is an effective tool to tune the membrane performance. Three isoreticular zeolitic inidazolate frameworks (ZIFs) and two matrix polymers (Matrimid and polybenzimidazole oPBI (PBI)) were used to prepare the MMMs. The ZIF linker functionality strongly determined the extent of the effect of PBI addition in the MMMs. For both the hydrophobic ZIF-7 and ZIF-8-based MMMs, PBI compatibilized the MOF-matrix interface and increased the $\mathrm{CO}_{2} / \mathrm{N}_{2}$ separation factor while slightly decreasing the permeability. Contrarily, the separation performance of the hydrophilic ZIF-90 MMM was not affected by PBI incorporation. Additionally, the MMM permeability followed the trend of the ZIF pore geometries and linker flexibilities. These results proved that MOF-matrix interfacial compatibility can effectively be controlled by polymer blending and that the extent of control is determined by a subtle balance between the MOF linker functionality and matrix chemistıy.
\end{abstract}

\section{Introduction}

Mixed matrix membranes (MMMs) consist of a (partially) inorganic additive phase dispersed in a continuous polymeric matrix [1]. For gas separation applications, these MMMs aim to synergistically combine the beneficial properties of both inorganic (highly permeable) membranes and polymeric (easily processable and selective) membranes [2]. Metal-organic frameworks (MOFs) are partially inorganic additives that have gained significant attention for MMMs. MOFs consist of metal cationic nodes linked by organic anions, resulting in crystalline nicroporous materials [3]. The attention for MOFs results from both the well-defined MOF crystal structure, in terms of pore aperture and cage size giving the MOFs molecular sieving properties and high sorption capacities towards specific gases, e.g., $\mathrm{CO}_{2}$ [3]. Moreover, in contrast with other inorganic MMM additives (e.g., zeolites), the use of MOFs in MMMs offers, due to the presence of the organic linker, a wide spectrum of tunability as well as enhanced matrix compatibility [1]
One subclass of MOFs that has gained significant attention due to their well-defined nicroporosity, functionality and thermal and chemical stability are zeolitic inidazolate frameworks (ZIFs) [4-6]. ZIFs, with network structures analogue to zeolites, are generally constructed by linking $\mathrm{Zn}^{2+}$ or $\mathrm{Co}^{2+}$ with inidazolate linkers, where the nature of the imidazolate determines the topology, pore dimensions and functionality of the framework. Interestingly, the high gas uptake of ZIFs arises from, in contrast with several other MOFs, the interaction between the gases and the inidazolate linkers and not from the metal sites [4]. Moreover, the use of different inidazolate linkers can result in the same framework topology, but with different pore dimensions. For example, an isoreticular sodalite (SOD) ZIF series can be obtained by using either, benzimidazole (bIm), 2-methylimidazole (mIn) or 2-imidazolecarboxaldehyde (Ica) as organic linker [7,8], resulting in ZIF-7, ZIF-8 and ZIF-90, respectively (Fig. 1). This isoreticular series shows also that the inidazolate linker not only deternines the size of the pore aperture and diameter (and consequently the crystal density and pore volune) but

\footnotetext{
$\because$ Colresponding author.

E-mail address: z.borneman@tue.nl (Z. Borneman).
} 


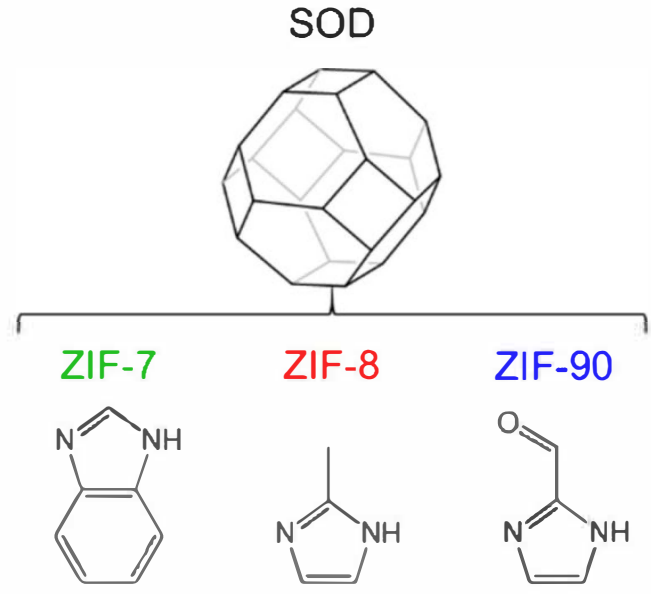

$\begin{array}{rcccl}\text { Linker } & b / m & m / m & \text { Ica } & \\ d_{a} & 2.9 & 3.4 & 3.5 & (\AA) \\ d_{p} & 4.3 & 11.6 & 11.2 & (\AA) \\ \rho_{c} & 1.24 & 1.14 & 0.99 & \left(\mathrm{~g} / \mathrm{cm}^{3}\right) \\ V_{p} & 0.21 & 0.49 & 0.58 & \left(\mathrm{~cm}^{3} / \mathrm{g}\right) \\ \mu_{1} & 1.38 & 1.25 & 2.87 & (D)\end{array}$

Fig. 1. Schematic overview of the isoreticular SOD series of ZIF-7, ZIF-8 and ZIF-90 with their corresponding organic linker, pore aperture $\left(d_{a}\right)$ and pore diameter $\left(d_{p}\right)$, crystal density $\left(\rho_{c}\right)$, pore volume $\left(V_{p}\right)$ and linker dipole moment $\left(\mu_{1}\right)[7-10]$.

also the pore functionality and polarity, as can be seen by the difference in molecular linker structure of mIn (methyl functionality) and Ica (aldehyde functionality).

ZIFs have been applied in gas separation membrane applications both as free standing membranes and in MMMs, where the majority of these membranes is based on ZIF-8 and to a lesser extent on ZIF-7 and ZIF-90 [5,6,11]. Due to the different size of the apertures of these ZIFs (Fig. 1), ZIF-7 is primarily used for hydrogen-based separations (kinetic diameter $\mathrm{H}_{2}=2.9 \AA$ ) and ZIF-8 and ZIF-90 are primarily used for $\mathrm{CO}_{2}$-based separations (kinetic diameter $\mathrm{CO}_{2}=3.3 \AA$ ) in MMMs. Generally, the incorporation of these ZIFs in polymers results in increased permeabilities and, depending on the type of separation and polymer/ZIF system, also in increased selectivities [5]. In particular, the use of ZIFs in MMMs is attractive for gas pairs where the size of the ZIF pore aperture lies in between the two kinetic diameters of the gases, facilitating highly permeable pathways for the gas with the smaller kinetic diameter while limiting the permeation of the gas with the bigger kinetic diameter [5].

Typical polyners used as matrices for ZIF-based MMMs are (fluorinated) polyimides, polyether-block-anides and polybenzinidazoles [5, 11]. The polyinide Matrimid® 5218 (Matrimid) can be regarded as a benchmark polymer for $\mathrm{CO}_{2}$ separations. Especially ZIF-8 is most commonly used as ZIF additive in Matrinid-based MMMs and has shown significant improvements on the MMM gas separation performances [12-17]. From a structural point of view, polybenzinidazoles are an interesting polyner class for ZIF-based MMMs as well, since the benzinidazole repeating unit is (partially) structurally analogous to the inidazole linkers of ZIFs. Thus far, from the many possible polybenzimidazole structures $m$-PBI has mainly been investigated as matrix for ZIF/polybenzimidazole MMMs. The incorporation of ZIF-7, ZIF-8 and ZIF-90 in m-PBI enhanced the $\mathrm{H}_{2} / \mathrm{CO}_{2}$ membrane separation performances, but, more importantly, showed excellent MOF-matrix compatibilities, since ZIF loadings higher than $50 \mathrm{wt} \%$ still resulted in defect free MMMs [18-22]. However, due to its rigid polyner chain packing, $m$-PBI is rather inpermeable for gases with a kinetic diameter bigger than $\mathrm{H}_{2}$ (e.g., a $\mathrm{CO}_{2}$ permeability lower than 0.17 Barrer) [21, 23]. Following the resistances in series model, the polymer matrix phase dominates the overall MMM permeability significantly decreasing the effectiveness of the highly permeable nature of MOFs. Therefore, the application of m-PBI-based as matrix for MMMs is linited to $\mathrm{H}_{2}$-based separations.

Despite the proven enhancenent of the separation performance of MOFs/ZIFs in MMMs, several challenges towards actual industrial implementation lie ahead in the field of MOF-based MMMs [2,5]. According to Lin et al., characterization of the interfacial interactions, defect formation, gas transport mechanism and mechanical stability are four key aspects in MOF-based MMMs that require systematic assessment to reach practical applications [24]. Regarding these key aspects, significant progress has been made in tailoring the properties of both ZIFs and matrices. For example, interfacial compatibility [14,25], interfacial sorption behavior [26], ZIF dispersion stability [27] and synergistic behavior between ZIFs and other additives [12,28] were proven to be important design parameters for ZIF-based MMMs. In addition, the importance of the ZIF-matrix compatibility was recently confirmed by molecular dynamic simulations by Ozcan et al. who proved that a poor ZIF-based MMM performance stems from poor compatibility between the ZIF and the matrix [29]. However, the majority of studies on the investigation of the previously mentioned key aspects focuses on one type of ZIF in combination with one type of polymer matrix material only, restricting findings to particular systems and liniting the development of a broader application framework [4,5, $11]$.

To gain a broader understanding of the nature and importance of these MOF-matrix interactions and their impact on actual membrane performance, this research systematically investigates how the addition of a polybenzinidazole to ZIF/Matrinid MMMs affects the $\mathrm{CO}_{2} / \mathrm{N}_{2}$ membrane separation performance. Matrinid is selected because of its benchmark status in literature, whereas polybenzimidazole is selected for its structural similarity with the ZIF linkers. Three isoreticular SOD ZIFs (ZIF-7, ZIF-8 and ZIF-90) with different pore apertures, diameters and functionalities are incorporated in the MMMs. With this approach it is possible to systenatically analyze to what extent polybenzinidazole and the ZIF linker functionality affect the ZIF-matrix interface and consequentially the membrane separation performance. In addition, the use of an isoreticular ZIF series also enables a systematic investigation of the influence of the different ZIF properties on the MMM separation performance.

\section{Materials and methods}

\subsection{Chemicals}

Benzimidazole (ZIF-7 linker, bIm, $97 \%$ purity) was purchased from Fluorochen Ltd (Hadfield, United Kingdom). 2-Methylimidazole (ZIF-8 linker, mIn, $99 \%$ purity), zinc acetate dihydrate $\left(\mathrm{Zn}(\mathrm{Ac})_{2} \cdot 2 \mathrm{H}_{2} \mathrm{O}\right.$, > $99.5 \%$ purity), N,N-dimethylformanide (DMF, $\geq 99.9 \%$ purity) and tetrahydrofuran (THF, $\geq 99.0 \%$ purity, 250 ppm BHT inhibitor) were purchased from Signa-Aldrich (Zwijndrecht, The Netherlands). 2-Inidazolecaboxaldehyde (ZIF-90 linker, Ica, $98 \%$ purity) was purchased from TCI (Zwijndrecht, Belgium). Matrimid® 5218 (polyinide, referred to as 'Matrimid') was kindly provided by Huntsman (Basel, Switzerland) and Fumion® AM (the polybenzinidazole oPBI, referred to as 'PBI') was purchased from Funatech (Bietigheim-Bissingen, Germany) (Fig. 2). This specific polybenzinidazole was selected as it is a relatively easy processable polybenzimidazole (by means of solubility) due to the rotatable/flexible biphenylether moiety [30]. Additionally, since Kumbharkar et al. showed that increasing the PBI flexibility by 
<smiles>CN1C(=O)c2ccc(C(=O)c3ccc4c(c3)C(=O)N(c3ccc(C5(C)CC(C)(C)c6ccccc65)cc3)C4=O)cc2C1=O</smiles>

Matrimid

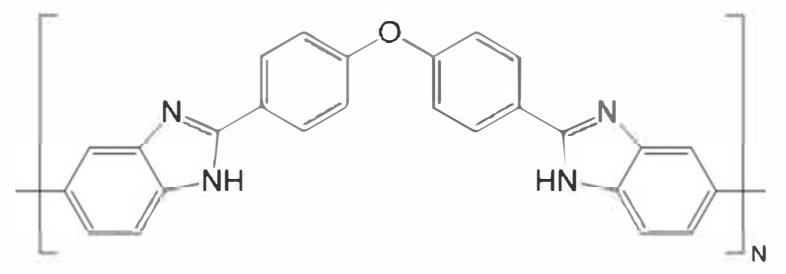

PBI

Fig. 2. Structural formula of Matrinid and PBI.

structural modification resulted in enhanced permeabilities (up to two orders of magnitude) [23,31], it is expected that incorporating oPBI in Matrinid matrices will have a minimal impact on the separation performance, relative to the pure Matrimid membrane. For sorption measurements, the gases helium (5.0 grade), nitrogen (4.5 grade) and carbon dioxide (4.5 grade) were purchased from Linde gas (Schiedam, The Netherlands). For permeation measurements, the gases nitrogen (5.0 grade), and carbon dioxide (5.0 grade) were purchased from Air Liquide (Herenthout, Belgiun). All chenicals were used as received.

\subsection{SOD ZIF syntheses}

For the ZIF syntheses 24 mmol linker (ZIF-7: $2.84 \mathrm{~g}$ bIn, ZIF-8: $1.97 \mathrm{~g}$ nIIn, ZIF-90: $2.30 \mathrm{~g}$ Ica) was dissolved in $60 \mathrm{~mL}$ DMF. The ZIF-90 linker suspension was heated to $100{ }^{\circ} \mathrm{C}$ for $1 \mathrm{~h}$ to completely dissolve the Ica. Meanwhile, three solutions of $3 \mathrm{mmol} \mathrm{Zn(Ac)})_{2} \cdot 2 \mathrm{H}_{2} \mathrm{O}(0.66 \mathrm{~g})$ in $15 \mathrm{~mL}$ DMF were prepared (one for each ZIF linker solution). These solutions were each added to the ZIF linker solutions and the synthesis mixtures were stirred for $1 \mathrm{~h}$. The resulting ZIF suspensions were centrifuged with a VWR CompactStar CS 4 for 10 min at $6500 \mathrm{rpm}$ and washed with DMF three times and once with THF. All steps were performed at room tenperature unless stated differently. The ZIFs were dried in a vacuum oven at 200 mbar at $70^{\circ} \mathrm{C}$ for $24 \mathrm{~h}$. Lastly, the ZIFs were further dried in a vacuum oven at $1 \mathrm{mbar}$ at $150^{\circ} \mathrm{C}$ for $12 \mathrm{~h}$.

\subsection{Membrane formation}

\subsubsection{Membrane compositions}

Pure polymer membranes and MMMs with 0, 12.5 and 25 wt\% PBI relative to the amount of Matrinid were formulated according to the compositions shown in Table 1 and Table 2, respectively. Significant enhancements in perneation behavior of ZIF-based MMMs have been reported for additive contents higher than $10 \mathrm{wt} \%[15,17,32]$. On the other hand, ZIF-8 loads equal to and higher than $30 \mathrm{wt} \%$ have shown losses in selectivity, implying defect formation in the MMMs $[16,17]$. Therefore, the MMM ZIF content was set at $20 \mathrm{wt} \%$ relative to the

Table 1

Overview of the compositions of the pure polymer menbranes.

\begin{tabular}{|c|c|c|c|}
\hline \multicolumn{2}{|c|}{ Matrimid } & \multicolumn{2}{|l|}{ PBI } \\
\hline (g) & $(\%)$ & (g) & $(\%)$ \\
\hline 0.420 & 100 & - & 0 \\
\hline 0.3675 & 87.5 & 0.0525 & 12.5 \\
\hline 0.315 & 75 & 0.105 & 25 \\
\hline
\end{tabular}

Table 2

Overview of the compositions of the MMMs.

\begin{tabular}{|c|c|c|c|c|c|c|c|}
\hline \multicolumn{6}{|l|}{ Matrix } & \multicolumn{2}{|c|}{ Additive } \\
\hline \multicolumn{2}{|c|}{ Matrimid } & \multicolumn{2}{|l|}{ PBI } & \multicolumn{2}{|c|}{ Total polymer } & \multicolumn{2}{|c|}{ ZIF-7, ZIF-8 or ZIF-90 } \\
\hline (g) & $(\%)$ & (g) & $(\%)$ & $(g)$ & $(\%)$ & (g) & (\%) \\
\hline 0.420 & 100 & - & 0 & 0.42 & 80 & 0.105 & 20 \\
\hline 0.3675 & 87.5 & 0.0525 & 12.5 & 0.42 & 80 & 0.105 & 20 \\
\hline 0.315 & 75 & 0.105 & 25 & 0.42 & 80 & 0.105 & 20 \\
\hline
\end{tabular}

polymer matrix, ensuring performance enhancement, but minimizing the chance of defect formation.

\subsubsection{Pure polymer membrane preparation}

First the desired amount of polymer (Table 1) was dissolved in a mixture of $2.4 \mathrm{~g}$ DMF and $2.4 \mathrm{~g}$ THF by continuous stirring for three days. After that, the polymer solutions were poured in Teflon dishes $(\mathrm{d}$ $=6 \mathrm{~cm}$ ) that were subsequently placed in an $\mathrm{N}_{2}$ atmosphere where the majority of solvent was evaporated during 5 days. After removing the membranes from the dishes by hand, the obtained membranes were further dried in an $\mathrm{N}_{2}$ oven at $80{ }^{\circ} \mathrm{C}$ for 2 days. Prior to the permeation measurements, circular membrane samples with a diameter of $20 \mathrm{~mm}$ were stamped that were thoroughly dried and annealed in a muffle oven by heating the coupons to $110^{\circ} \mathrm{C}$ (heating rate $=5^{\circ} \mathrm{C} / \mathrm{min}$ ), holding this temperature for $2 \mathrm{~h}$, increasing the temperature to $180{ }^{\circ} \mathrm{C}$ (heating rate $=5{ }^{\circ} \mathrm{C} / \mathrm{min}$ ) and holding the temperature for another $6 \mathrm{~h}$. The samples were stored at room temperature for 1-3 days before the permeation measurement.

\subsubsection{ZIF-7 and ZIF-8 MMM preparation}

Either $0.105 \mathrm{~g}$ ZIF-7 or $0.105 \mathrm{~g}$ ZIF-8 was dispersed in a mixture of $2.4 \mathrm{~g}$ DMF and $2.4 \mathrm{~g}$ THF by sonicating the suspension in a Branson 3510 sonication bath for 30 min. If the MMM contained PBI, depending on the polymer matrix composition (Table 2), PBI was added to the suspension and the mixture was stirred for two days. Then, Matrinid was added and the mixture was stirred for another two days. The ZIF-7/polymer and ZIF-8/polymer mixtures were subsequently cast, dried and annealed following the same protocol as for the pure polymer membranes resulting in membrane samples with 20 wt\% additives.

\subsubsection{ZIF-90 MMM preparation}

As the fabrication of the ZIF-90 MMMs following the protocol used for the ZIF-7 and ZIF-8 MMM syntheses resulted in gelation of the casting solutions, a separate protocol for the ZIF-90 MMMs is given. Pure Matrimid and Matrinid/PBI compositions as shown in Table 2 were dissolved in a mixture of $2.4 \mathrm{~g}$ DMF and $2.4 \mathrm{~g}$ THF by continuous stirring for three days. Then, $0.105 \mathrm{~g}$ ZIF-90 was dispersed in $2.4 \mathrm{~g}$ DMF and 2.4 $\mathrm{g}$ THF by sonicating the suspension in a Branson 3510 sonication bath for 30 min. Prior to casting, the ZIF-90 suspensions were added to the polymer solutions and the mixtures were briefly stirred. The ZIF-90/ polymer mixtures were subsequently cast, dried and annealed following the same protocol as for the pure polymer nembranes resulting in membrane samples with $20 \mathrm{wt} \%$ additives.

\subsection{Characterization}

X-ray diffraction (XRD) patterns of the ZIFs were obtained with a Rigaku Miniflex $600\left(15 \mathrm{~mA}, 40 \mathrm{kV}, \mathrm{Cu} \mathrm{K}_{\alpha}\right.$ radiation $(\lambda=1.5406 \AA)$ ) with $2 \theta$ ranging from $5^{\circ}$ to $50^{\circ}$ and a scanning rate of $2^{\circ} / \mathrm{min}$.

The Brunauer-Enmett-Teller (BET) surface area of the ZIFs was determined by $\mathrm{N}_{2}$ physisorption with a Micromeretics Tristar II (liquid $\mathrm{N}_{2},-196^{\circ} \mathrm{C}$ ). Prior to the measurement, the samples were dried in a vacuum oven at $150^{\circ} \mathrm{C}$ and $1 \mathrm{mbar}$ for $24 \mathrm{~h}$.

The thermal stability of the ZIFs, the pure membranes and the MMMs was analyzed with a TA Instruments TGA Q500 under an inert $\mathrm{N}_{2}$ 
atmosphere. In the first temperature range, the temperature was increased from 50 to $400{ }^{\circ} \mathrm{C}$ with $10^{\circ} \mathrm{C} / \mathrm{min}$. In the second temperature range, the temperature was increased from 400 to 900 with $5{ }^{\circ} \mathrm{C} / \mathrm{min}$ to increase the resolution during the degradation of organic compounds.

The glass transition temperature $\left(\mathrm{T}_{\mathrm{g}}\right)$ of $\mathrm{PBI}$, the pure membranes and the MMMs was determined by differential scanning calorimetry (DSC) with a TA Instruments DSC Q2000. Two temperature cycles from $0{ }^{\circ} \mathrm{C}$ to $450{ }^{\circ} \mathrm{C}$ with a heating/cooling rate of $20^{\circ} \mathrm{C} /$ min were applied and the $\mathrm{T}_{\mathrm{g}}$ was determined from the mid-point temperature of the phase transition in the second heating cycle.

The morphology of the ZIFs and the cross-sections of all membrane types were analyzed with a JEOL JSM-IT100 scanning electron microscope (SEM). Prior to the analysis, the membranes were wetted in a 50:50 water/isopropanol mixture and cryogenically fractured. Then, all samples were gold sputter coated with a JEOL JFC-2300 HR sputter coater to prevent sample charging.

The elemental distribution of carbon and zinc in the MMMs containing the ZIF particles was analyzed by energy dispersive X-ray spectroscopy (EDS, JEOL JSM-IT100) where the probe current was set to an intensity such that $\pm 1-1.310^{4}$ counts per second were registered. Crosssections of the MMMs for the EDS analysis were prepared following the same protocol as the preparation of the membrane samples for the SEM analysis.

High pressure $\mathrm{N}_{2}$ and $\mathrm{CO}_{2}$ sorption of the ZIFs, PBI, the pure membranes and the MMMs was measured with a Rubotherm series IsoSORP $\mathbb{B}$ sorption balance at pressures ranging from 1 to 15 bar at $35^{\circ} \mathrm{C}$. All samples were degassed at vacuum until a stable weight was obtained before each sorption measurement. Measurements of the membrane samples were performed by loading the balance with the membrane coupons used for permeation measurements all at once. This was done to increase the accuracy of the measurement as the resolution of the sorption balance is $10 \mu \mathrm{g}$ and the range of gas uptake of the membranes is in the order of $50-500 \mu \mathrm{g}$ when this protocol is applied (depending on the membrane type and the penetrant. This resulted in the limitation of only one sorption measurement per membrane type but significantly improved the reliability of the obtained data. Additionally, to limit the effect of physical aging for the membrane samples, all solubilities were determined within a time span of three weeks (the minimal time required to measure the solubility of all samples). Prior to the $\mathrm{N}_{2}$ and $\mathrm{CO}_{2}$ sorption measurements, the density of the samples was determined by helium pycnometry. The measured weight increase of the samples was corrected according to Archimedes' principle (Equation (1)). In Equation (1) $\mathrm{m}_{\text {corrected }}$ is the corrected weight $(\mathrm{g}), \mathrm{m}_{\text {measured }}$ the measured weight $(\mathrm{g}), \rho_{\text {gas }}$ the density of the measuring gas $\left(\mathrm{g} / \mathrm{cm}^{3}\right)$ and $\mathrm{V}_{\text {sample }}$ the sample volume $\left(\mathrm{cm}^{3}\right)$. The measured sorption values were divided by the corresponding pressure (in $\mathrm{cmHg}$ ) to obtain the solubility.

$m_{\text {corrected }}=m_{\text {measured }}+\rho_{\text {gas }} \cdot V_{\text {sample }}$

Mixed gas $\mathrm{CO}_{2} / \mathrm{N}_{2}$ perneation experiments were performed with a high-throughput gas separation system [33,34]. This system enables accurate mixed gas permeation measurements of maximum 16 membranes simultaneously. The $\mathrm{CO}_{2} / \mathrm{N}_{2}$ feed was mixed in a 50:50 composition at 10 bar feed pressure and $35^{\circ} \mathrm{C}$, where the feed flow rate was significantly higher than the permeate flow rate to ensure a constant feed composition. Prior to the permeation measurements, the menbranes were conditioned for at least $12 \mathrm{~h}$. The permeate composition was determined with an Interscience gas chromatograph. The separation factor was determined according to Equation (2), where $\alpha_{\mathrm{CO} 2 / \mathrm{N} 2}$ is the $\mathrm{CO}_{2} / \mathrm{N}_{2}$ separation factor $(-), \mathrm{y}_{\mathrm{CO} 2}$ the $\mathrm{CO}_{2}$ permeate fraction $(-), \mathrm{y}_{\mathrm{N} 2}$ the $\mathrm{N}_{2}$ permeate fraction $(-), \mathrm{x}_{\mathrm{CO} 2}$ the $\mathrm{CO}_{2}$ feed fraction $(-)$ and $\mathrm{x}_{\mathrm{N} 2}$ the $\mathrm{N}_{2}$ feed fraction (-), $\mathrm{P}_{\mathrm{CO} 2}$ the mixed gas $\mathrm{CO}_{2}$ permeability (Barrer) and $\mathrm{P}_{\mathrm{N} 2}$ the mixed gas $\mathrm{N}_{2}$ permeability (Barrer).

$\alpha_{\mathrm{CO}_{2} / \mathrm{N}_{2}}=\frac{y_{\mathrm{CO}_{2}} / y_{N_{2}}}{x_{\mathrm{CO}_{2}} / x_{N_{2}}}=\frac{P_{\mathrm{CO}_{2}}}{P_{\mathrm{N}_{2}}}$
The overall membrane permeability, i.e., the mixed gas permeability of $\mathrm{N}_{2}$ and $\mathrm{CO}_{2}$ combined, was determined by measuring the increase in permeate pressure over time in a calibrated volume and is calculated according to Equation (3), where $P_{\text {total }}$ is the overall permeability (Barrer), $\Delta$ ppermeate the increase in permeate pressure $(\mathrm{Pa}), \mathrm{V}_{\mathrm{c}}$ the calibrated permeate volume $\left(\mathrm{m}^{3}\right), \mathrm{V}_{\mathrm{m}}$ the molar volume at STP $\left(\mathrm{cm}^{3} / \mathrm{mol}\right), \mathrm{L}$ the membrane thickness $(\mathrm{cm}), \Delta t$ the time interval (s), $R$ the gas constant $(\mathrm{J} /(\mathrm{K} \cdot \mathrm{mol}))$, $\mathrm{T}$ the permeate temperature $(\mathrm{K}), \mathrm{A}$ the membrane area $\left(\mathrm{cm}^{2}\right)$ and $\Delta \mathrm{p}$ the transmembrane pressure $(\mathrm{cmHg})$.

$P_{\text {total }}=\frac{\Delta p_{\text {permeate }} \cdot V_{c} \cdot V_{m} \cdot L \cdot 10^{10}}{\Delta t \cdot R \cdot T \cdot A \cdot \Delta p}$

The mixed gas $\mathrm{CO}_{2}$ and $\mathrm{N}_{2}$ permeabilities were obtained by combining Equation (2) and Equation (4).

$P_{\text {total }}=x_{i} \cdot P_{i}+x_{j} \cdot P_{j}$

The diffusivities were calculated using Equation (5), where D is the diffusivity $\left(\mathrm{cm}^{2} / \mathrm{s}\right), \mathrm{P}$ the permeability (Barrer) and $\mathrm{S}$ the solubility coefficient $\left(\mathrm{cm}^{3}(\mathrm{STP}) /\left(\mathrm{cm}^{3} \cdot \mathrm{cmlHg}\right)\right)$. Since the permeability was measured at 10 bar feed pressure, the sorption quantities of the membranes of both $\mathrm{N}_{2}$ and $\mathrm{CO}_{2}$ at 5 bar (the effective partial pressure for a 50:50 mixed gas at 10 bar) were normalized for the pressure to obtain the solubility coefficients.

$D=P / S$

The diffusivities are thus calculated using the mixed gas permeability but single gas sorption values, neglecting the possibility of competitive sorption during sorption measurements $[35,36]$. Moreover, in sorption experiments the surrounding gas pressure is everywhere the same, where in the permeability measurements the upstrean gas pressure is significantly higher than the downstream pressure (vacuum). Therefore, the diffusivity values are used to visualize and analyze trends rather than reporting absolute values. In addition to the absolute diffusivities, relative membrane diffusivities were calculated by dividing the membrane diffusivity by the diffusivity of the respective membrane type without PBI incorporation to facilitate the analysis of the effect of PBI incorporation on the membrane diffusivities relative to that of the membranes without PBI.

\section{Results and discussion}

\subsection{Particle analysis}

The XRD patterns of ZIF-7, ZIF-8 and ZIF-90 are shown in Figure S1. The diffraction patterns of all three ZIFs are in agreement with the patterns reported in literature, confirming the presence of the intended crystalline structures $[7,8]$.

The thermal stability of the ZIF particles is shown in Figure S2. From the three ZIFs, ZIF-7 shows the highest onset in degradation temperature $\left( \pm 500{ }^{\circ} \mathrm{C}\right.$ ), followed by ZIF-8 (onset degradation temperature is $\pm 400^{\circ} \mathrm{C}$ ) and ZIF-90 (onset degradation temperature is $\pm 280^{\circ} \mathrm{C}$ ), which corresponds with literature $[7,8,37]$. For all ZIFs, the weight loss after the degradation onset is attributed to the carbonization of the ZIF linkers [38]. The differences in residual weight at $900{ }^{\circ} \mathrm{C}$ between the ZIFs are in agreement with literature as well, where ZIF-7 with the highest molecular weight linker has the lowest relative residual weight after linker carbonization [7,8,20,38].

The $\mathrm{N}_{2}$ physisorption isotherms and BET surface areas of the ZIFs are shown in Figure S3. All ZIFs show type I adsorption isotherms that are typical for microporous solids and have a BET surface area that is within the range of reported literature values $[7,8,16,39-43]$. In addition, the upswing in $\mathrm{N}_{2}$ adsorption at $\mathrm{p} / \mathrm{p}_{0} \approx 1$ of all ZIFs is typical for microporous nano-sized materials due multilayer adsorption at the interparticle space between external surfaces $[39,44]$.

SEM analysis of the ZIFs shows that the used synthesis protocols 
result in submicron-sized particles (Fig. 3). The ZIF particles with isotropic particle morphologies have diameters of approxinnately $60 \mathrm{~nm}$, $120 \mathrm{~nm}$ and $85 \mathrm{~nm}$, for ZIF-7, ZIF-8 and ZIF-90, respectively. This submicron particle size range is typical for ZIF syntheses that use high inidazole: $\mathrm{Zn}^{2+}$ ratios and is assumed to be caused by increased nucleation rates $[45,46]$. As subnicron-sized ZIFs have shown improved separation performances relative to micron-sized ZIFs in MMMs, especially within the range of $100 \mathrm{~nm}$, the obtained ZIF particle sizes are optinal for application in MMMs [19,47,48].

The high pressure $\mathrm{CO}_{2}$ and $\mathrm{N}_{2}$ sorption isotherms of the ZIFs are shown in Fig. 4.

Clearly, the $\mathrm{CO}_{2}$ sorption is higher than the $\mathrm{N}_{2}$ sorption for all ZIFs. This difference in gas uptake between the two gases in the ZIFs is attributed to their difference in quadrupole moment. $\mathrm{CO}_{2}$ has both stronger interactions with the inidazolate groups and is more condensable than $\mathrm{N}_{2}$, due to its significantly bigger quadrupole moment $\left(-14.27 \cdot 10^{-40}\right.$ and $-4.65 \cdot 10^{-40} \mathrm{C} \mathrm{m}^{2}$, respectively) $[4,49]$. For both gas types the order of gas uptake is ZIF-7 $<$ ZIF-90 $<$ ZIF-8. ZIF-7 exhibits the lowest sorption capacity due to its small pore volume (at least \pm 2.5 times smaller than the ZIF-8 and ZIF-90 pore volumes (Fig. 1) and narrow crystallographic pore aperture (at least 0.5 A snialler than ZIF-8 and ZIF-90) [9]. Although ZIF-7 should not be accessible towards gas with a kinetic dianeter bigger than the ZIF-7 pore aperture (2.9 $\AA$ ), the fact that ZIF-7 sorbs $\mathrm{CO}_{2}$ and to a lesser extent $\mathrm{N}_{2}$ is attributed to the penetrant induced gate opening mechanism that is present in ZIF-7 [50, 51], where the gate opening effect occurs at significantly lower $\mathrm{CO}_{2}$ pressures than $\mathrm{N}_{2}$ pressures [52]. Interestingly, ZIF-8 and ZIF-90 show very comparable uptakes of $\mathrm{N}_{2}$ and $\mathrm{CO}_{2}$ at low pressures, with the $\mathrm{CO}_{2}$ values being an order of magnitude higher than those of $\mathrm{N}_{2}$, but differ significantly at high gas pressures, despite their comparable crystallographic pores sizes and apertures (Fig. 1). Up to 2 bar, due to its polar functionality ZIF-90 shows a higher $\mathrm{CO}_{2}$ uptake than ZIF-8 (51\% at 1 bar and $15 \%$ at 2 bar) [53,54]. When the $\mathrm{CO}_{2}$ pressure is increased, ZIF-8 surpasses the ZIF-90 $\mathrm{CO}_{2}$ uptake, which is ascribed to the difference in linkers. The ZIF-8 linker, mIm, shows, in comparison with the ZIF-90 linker, Ica, a significantly higher rotatability. This 'swinging motion' of the linker results in dynanic larger pore apertures that increase the overall uptake capacity of gases in the ZIF structure $[55,56]$. This effect of the linker flexibility on the sorption capacity also explains the higher $\mathrm{N}_{2}$ uptake of ZIF-8 relative to ZIF-90 at higher pressures.

\subsection{Membrane analysis}

The thermal stability of the pure polymer membranes and the ZIF containing MMMs is shown in Figure S4. All membranes show a very small decrease in weight at $100{ }^{\circ} \mathrm{C}$ due to the release of water. This decrease is stronger for the ZIF-90 MMMs (Figure S4 D) due the hydrophilic nature of ZIF-90 [57]. Both the pure polymer membranes and the ZIF MMMs show a first onset in degradation temperature at approxinately $300{ }^{\circ} \mathrm{C}$ followed by a second onset in degradation tenperature at approximately $450{ }^{\circ} \mathrm{C}$. The first onset is attributed to the release of entrapped guest molecules, e.g., solvent, which are released more readily once the temperature approaches the $\mathrm{T}_{g}$ of the matrix [32]. The second onset is attributed to the carbonization of the polymer

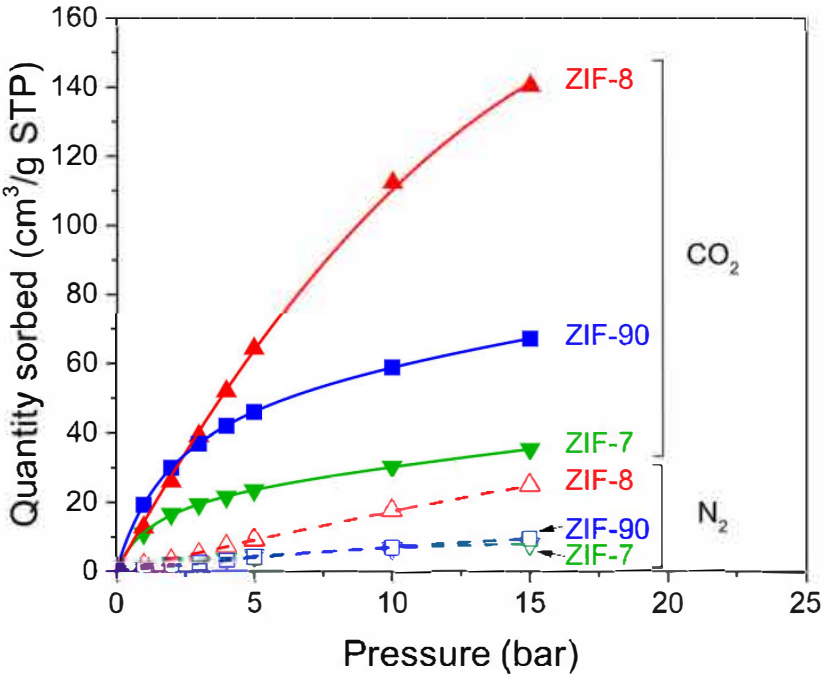

Fig. 4. $\mathrm{CO}_{2}$ (solid lines, closed symbols) and $\mathrm{N}_{2}$ (dashed lines, open symbols) high pressure sorption isotherms at $35^{\circ} \mathrm{C}$ of ZIF-7 (triangle down, green), ZIF-8 (triangle up, red) and ZIF-90 (square, blue).

matrix and, if present, of the ZIFs [32]. Increasing the amount of PBI in the membranes consistently results in higher residual weights, showing that PBI exceeds the thermal stability of Matrinid.

Next, the glass transition temperatures of the pure polymer menbranes and the ZIF MMMs are shown in Table 3 . The $\mathrm{T}_{\mathrm{g}}$ of Matrinid $(0 \%$ PBI) closely matches the values found in literature $\left(302-328^{\circ} \mathrm{C}[15\right.$, 58]). Relative to the conventional $m$-PBI $\left(\mathrm{T}_{g}=416^{\circ} \mathrm{C}\right.$ [23]), the PBI used in this work (oPBI, $\mathrm{T}_{g}=315^{\circ} \mathrm{C}$ ) has a significantly lower glass transition temperature. This difference is caused by the difference in polyner structure of $m$-PBI and oPBI, where the latter has a significantly higher chain mobility due to its biphenylether moiety. Similar reductions in $\mathrm{T}_{g}$ were observed as well in systematically modified PBI series where an increased chain mobility resulted in a suppression of the $\mathrm{T}_{\mathbf{g}}[23,31]$. The incorporation of PBI in Matrinid does not significantly change the $T_{g}$ of the pure polymer membranes showing that the bulk interactions in the matrix remain unchanged. Within the range of accuracy of $\mathrm{T}_{g}$ deternination by DSC, these results closely match with the theoretical $\mathrm{T}_{\mathrm{g}}$ of the blends $\left(322{ }^{\circ} \mathrm{C}\right.$ and $321^{\circ} \mathrm{C}$ for $12.5 \mathrm{wt} \%$ and $25 \mathrm{wt} \% \mathrm{PBI}$, respectively) as

Table 3

Glass transition temperatures as determined by DSC of the pure polymer membranes and the ZIF MMMs (20 wt\% ZIF) all with •, 12.5 and $25 \%$ PBI content and pure PBI (100 wt \% PBI).

\begin{tabular}{lllll}
\hline \multirow{5}{*}{$\begin{array}{llll}\text { Membrane type } \\
\text { Pure polymer }\end{array}$} & ZIF-7 MMM & ZIF-8 MMM & ZIF-90 MMM \\
\hline wt.\% PBI & $T_{\boldsymbol{g}}\left({ }^{\circ} \mathrm{C}\right)$ & & & \\
0 & 323 & 334 & 330 & 322 \\
12.5 & 323 & 322 & 323 & 313 \\
25 & 322 & 317 & 320 & 311 \\
100 & 315 & & & \\
\hline
\end{tabular}
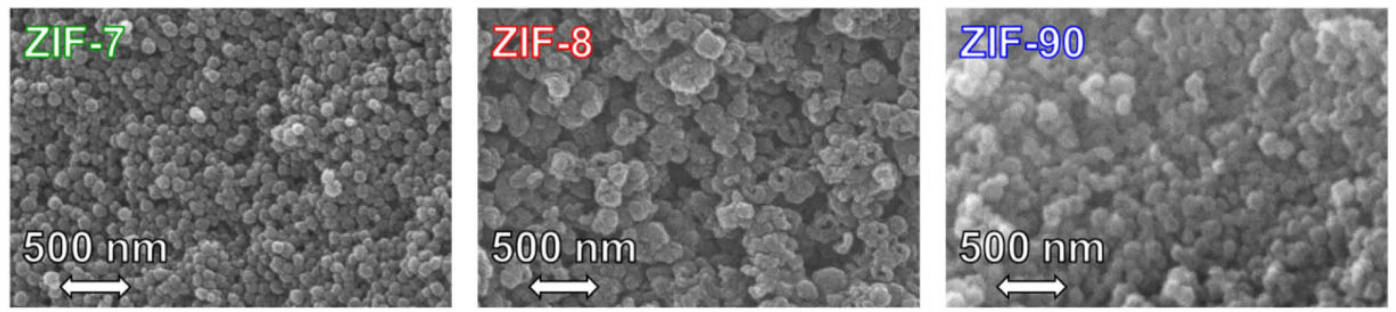

Fig. 3. SEM analysis of ZIF-7, ZIF-8 and ZIF-90. 
calculated by the Fox equation (Equation (6), where $\mathrm{W}$ is the polymer mass fraction of polymer 1 or 2) [59].

$\frac{1}{T_{g}}=\frac{W_{1}}{T_{g 1}}+\frac{W_{2}}{T_{g 2}}$

The incorporation of additives (e.g. ZIFs) in polymer materials can affect the $\mathrm{T}_{g}$ of the composite material in three manners: (1) strong interaction between the additive and the matrix decreases the interfacial polymer mobility (rigidification/physical crosslinking), enhancing the $\mathrm{T}_{g}$, (2) weak interaction between the additive and the matrix distorts the interfacial polymer mobility (derigidification), suppressing the $\mathrm{T}_{\mathrm{g}}$, or (3) the additive does not influence the polymer bulk arrangement, leaving the $\mathrm{T}_{\mathrm{g}}$ unchanged $[60,61]$. The addition of the ZIFs to Matrimid ( $\mathrm{wt} \%$ PBI) affects the MMM $\mathrm{T}_{\mathrm{g}}$ in two manners (Table 3). The $\mathrm{T}_{\mathrm{g}}$ of the ZIF-7 and ZIF-8 MMMs is enhanced, but the $\mathrm{T}_{\mathrm{g}}$ of the ZIF-90 MMMs remains approximately unchanged. This difference between the $T_{g}$ of ZIF-8/Matrimid and ZIF-90/Matrimid was observed in literature as well
$[15,61]$. These different effects of the ZIFs on the $T_{g}$ of the MMMs are attributed to difference in functionality of the ZIF linkers as the $T_{g}$ of MMMs qualitatively indicates changes in the MOF-matrix interface. Since the electron withdrawing aldehyde functionality of Ica (ZIF-90) distorts the electron delocalization in this $\mathrm{ZIF}, \pi-\pi$ interactions between the matrix and the linker will be less significant relative to the bIn (ZIF-7) and mIm (ZIF-8) linkers. As these $\pi$ - $\pi$ interactions were proposed to be a major contributing factor to rigidification of ZIF-8/Matrimid MMMs, the distorted electron delocalization of the ZIF-90 linkers and consequently the reduced interaction with Matrinid can therefore be used as an explanation that $T_{g}$ is not altered by the incorporation of ZIF-90 in Matrinid (Fig. 1) [62]. As this effect is not present in ZIF-7 and ZIF-8, $\pi-\pi$ interactions between these ZIFs and the polyner matrix do play a role, thus enhancing the $\mathrm{T}_{\mathrm{g}}$ of these two MMM types.

The addition of PBI to the MMMs clearly decreases the $\mathrm{T}_{\mathrm{g}}$ within each individual ZIF series relative to the specific pure polymer counterpart. When the different ZIF MMMs with a set amount of PBI are compared,

\section{A) Pure polymer membranes}
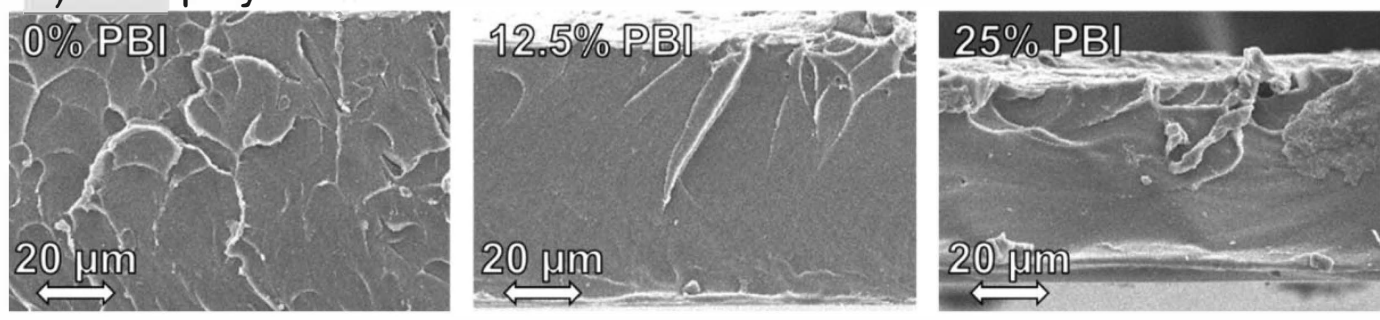

B) ZIF-7 MMMs
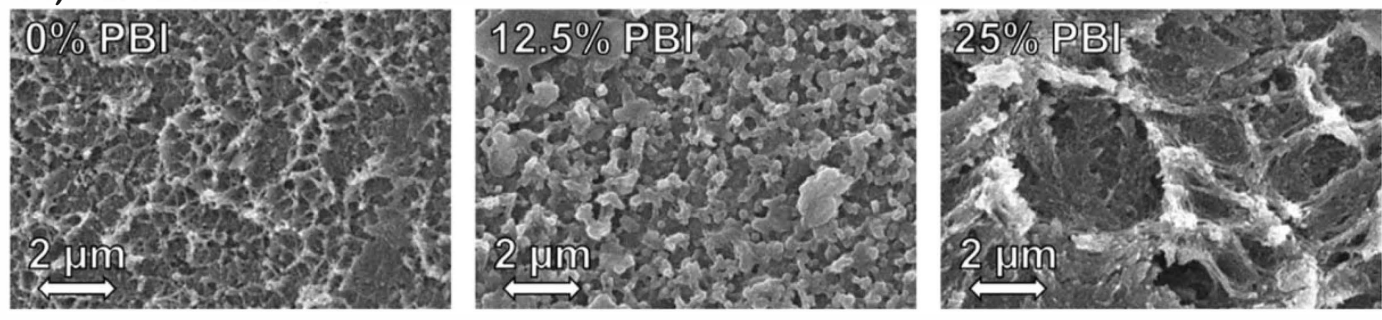

\section{C) ZIF-8 MMMs}
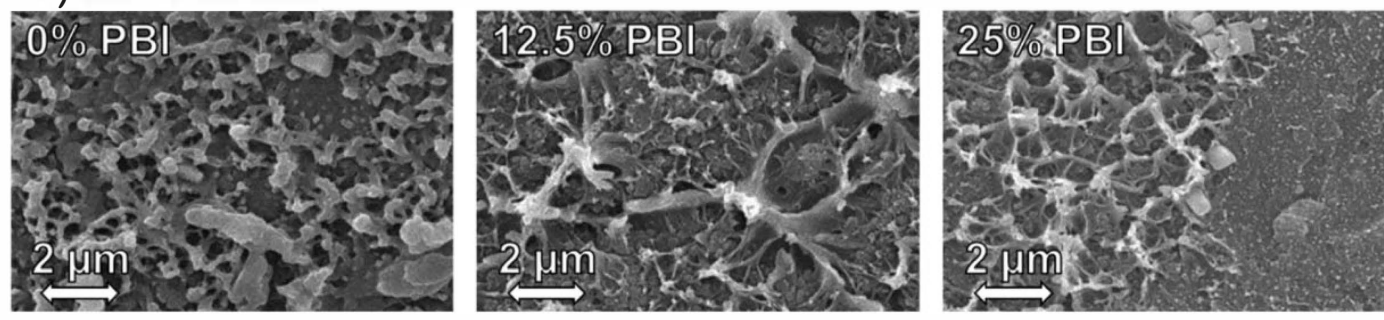

D) ZIF-90 MMMs
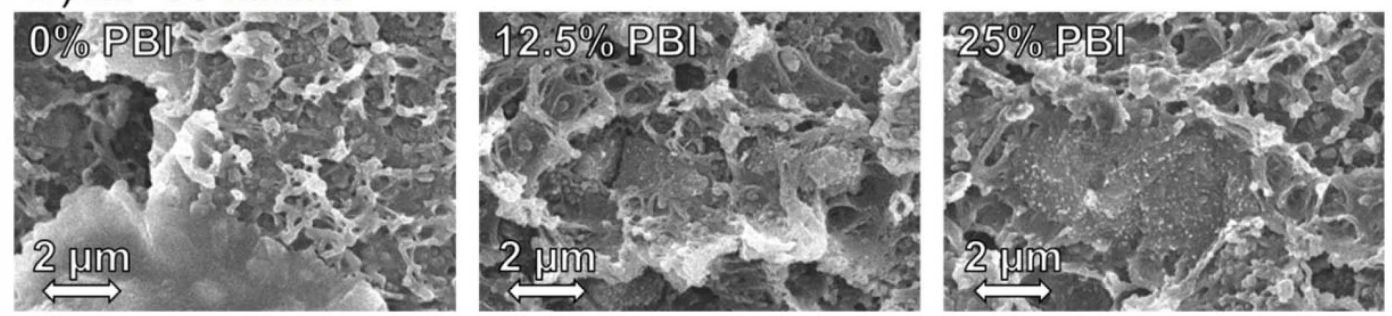

Fig. 5. SEM analysis of the cross-sections of A) Pure polymer membranes, B) ZIF-7 MMMs, C) ZIF-8 MMMs and D) ZIF-90 MMMs, all with 0 , 12.5 and $25 \%$ PBI content. 
different phenomena are observed. In that case, the addition of PBI af fects the $T_{g}$ of the materials in two ways. The ZIF-7 and ZIF-8 MMMs show a decrease in $T_{g}$ upon PBI incorporation, where the $T_{g}$ values lie between the $\mathrm{T}_{g}$ of pure Matrimid and PBI. This decrease indicates that ZIF-PBI interactions replace the ZIF-Matrimid interactions, since the rigidifying effect of the additives in the MMMs is diminished. Yang et al. confirmed this favorable ZIF-PBI interaction in ZIF-7/m-PBI MMMs where the amount of free $\mathrm{N}-\mathrm{H}$ bonds of $m$-PBI in ZIF-7/m-PBI MMMs decreased with increasing ZIF-7 content [20]. Oppositely, the ZIF-90 MMM shows $\mathrm{T}_{g}$ values that lie below the $\mathrm{T}_{g}$ of pure $\mathrm{PBI}$, indicating that, in contrast to the ZIF-7 and ZIF-8 MMMs, ZIF-90 distorts the packing of the polymer around the additive. On top of the previously mentioned distorted delocalization in the Ica linker, the electron withdrawing aldehyde functionality causes Ica to be more acidic than bIm and mIn, preventing proton transfer from PBI to the Ica linkers and consequently decreasing the interaction between $\mathrm{ZIF}-90$ and $\mathrm{PBI}$ resulting in lower $\mathrm{T}_{g}$ values.

SEM images of cross-sections of the pure polymer membranes and the ZIF-based MMMs are shown in Fig. 5. The pure polymer membrane cross-sections are smooth and defect free. When ZIFs are incorporated, the cross-sections show the typical crater-like structure of ZIF MMMs. Also it is clear that the individual ZIF particles are randomly distributed throughout the matrix. This random distribution is further confirmed by the EDS analysis of the MMMs (Figure S5, S6 and S7) where all samples show that the majority of zinc is homogeneously distributed throughout the matrices. In all MMMs several locations with ZIF particle sizes bigger than those of the pure ZIFs are observed (Fig. 3 vs Fig. 5), showing that the MMM casting method induces Ostwald ripening of the ZIF particles, i.e., the growth of larger particle by the recombination and redeposition of smaller particles [63]. This is in accordance with literature and does not deteriorate the membrane performance significantly [63].

\subsection{Membrane separation properties and performance}

\subsection{1. $\mathrm{N}_{2}$ and $\mathrm{CO}_{2}$ membrane solubility}

The $\mathrm{N}_{2}$ and $\mathrm{CO}_{2}$ solubility as function of the membrane PBI content in the pure polymer membranes and the ZIF MMMs at 5 bar and $35^{\circ} \mathrm{C}$ is shown in Fig. 6. The significant difference between $\mathrm{N}_{2}$ and $\mathrm{CO}_{2}$ uptake (both graphs have different Y-axis scales) of the membranes is attributed to their difference in critical temperature, which is related to solubility as function of the fluid condensability and the quadrupole moment, providing electrostatic affinity [64]. $\mathrm{CO}_{2}$ has both a higher critical temperature and a bigger quadrupole moment than $\mathrm{N}_{2}$ and is therefore significantly more sorbed in the polymer nembranes $[9,49]$.

The ZIF-based MMMs without PBI all show an increase in $\mathrm{N}_{2}$ solubility relative to the pure Matrinid membrane. Increasing the PBI content in the nembranes increases the $\mathrm{N}_{2}$ solubility of the pure polymer membranes and the ZIF-7 MMMs, but decreases the $\mathrm{N}_{2}$ solubility of the ZIF-8 and ZIF-90 MMMs. Since, absolutely, the $\mathrm{N}_{2}$ solubility in pure PBI at 5 bar $\left(1.2 \cdot 10^{-2} \mathrm{~cm}^{3} /\left(\mathrm{cm}^{3} \cdot \mathrm{cm} H g\right) \mathrm{STP}\right.$, not shown in Fig. $\left.6 \mathrm{~A}\right)$ is higher than that of Matrimid, it is expected that an increased $\mathrm{N}_{2}$ solubility should be observed for all membranes upon PBI incorporation. While this is not the case for the ZIF-8 and ZIF-90 MMMs, this indicates that for those MMMs PBI incorporation either intrudes the pores of these ZIFs or linits the previously mentioned linker rotatability of ZIF-8 and ZIF-90 lowering the $\mathrm{N}_{2}$ uptake, where either of these effects is especially present for ZIF-8 that has a significantly higher $\mathrm{N}_{2}$ uptake than ZIF-90 (Fig. 4). Since ZIF-7 has pore dimensions that are significantly smaller than that of ZIF-8 and ZIF-90, this decreased $\mathrm{N}_{2}$ solubility is not observed for the respective MMMs and PBI incorporation increases the $\mathrm{N}_{2}$ solubility as the matrix solubility is increased.

Oppositely, for the $\mathrm{CO}_{2}$ solubility in the membranes two clear trends can be distinguished. First, the incorporation of PBI in both the pure polyner membranes and the ZIF MMMs increases the membrane $\mathrm{CO}_{2}$ solubility (the $0 \mathrm{wt} \%$ PBI ZIF-7 MMM forms an exception as the $12.5 \mathrm{wt}$ \% ZIF-7 MMM has a lower $\mathrm{CO}_{2}$ solubility than the $0 \mathrm{wt} \%$ PBI ZIF-7 MMM). Comparing the $\mathrm{CO}_{2}$ sorption in pure Matrimid $\left(8.6 \cdot 10^{-2} \mathrm{~cm}^{3}\right.$ / $\left(\mathrm{cm}^{3} \cdot \mathrm{cm} H g\right)$ STP, Fig. $\left.6 \mathrm{~B}\right)$ with that in pure PBI $\left(13.2 \cdot 10^{-2} \mathrm{~cm}^{3} /\right.$ $\left(\mathrm{cm}^{3} \cdot \mathrm{cm} H g\right)$ STP, not shown in Fig. $\left.6 \mathrm{~B}\right)$ explains the increasing $\mathrm{CO}_{2}$ solubility of the pure polymer membranes and the MMMs with increasing PBI content since the $\mathrm{CO}_{2}$ solubility in PBI is significantly higher than the Matrimid $\mathrm{CO}_{2}$ solubility. Second, the influence of the size of the ZIF pore apertures and volumes and ZIF linker rotatability (Fig. 1) on the MMM sorption is clearly visible. The MMMs containing ZIF-8 and ZIF-90, which both have pore apertures that are accessible for $\mathrm{CO}_{2}$, show increased $\mathrm{CO}_{2}$ uptakes relative to the pure polymer membranes. Regardless of the PBI content, ZIF-8 increases the $\mathrm{MMM} \mathrm{CO}_{2}$ solubility with approximately $30-35 \%$ and ZIF-90 increases the MMM $\mathrm{CO}_{2}$ solubility with approximately $14 \%$, both relative to the pure polymer membranes. This increase in $\mathrm{CO}_{2}$ uptake is due to the high fractional void volume (the combination of the MOF fractional pore volume and the polymer fractional free volume) of the ZIF- 8 and ZIF-90 MMMs, as both ZIFs have a void volume that is roughly 2.5 times higher than the matrices $[65,66]$. However this does not support the fact that the ZIF-8 MMM CO 2 uptakes are consistently higher than the ZIF-90 $\mathrm{MMM} \mathrm{CO}_{2}$ uptakes. This discrepancy is attributed to the previously mentioned rotatability of the ZIF-8 linkers that facilitates a higher $\mathrm{CO}_{2}$ uptake in ZIF-8 than in ZIF-90 [55,56]. Contrary to the enhanced ZIF-8 and ZIF-90 MMM CO 2 solubilities, the incorporation of ZIF-7 in the matrices results in at maxinum a $\mathrm{CO}_{2}$ solubility equal to the pure polymer membrane, although the ZIF-7 fractional void volume is roughly 1.3 times higher than that of the matrices $[65,66]$. This behavior is caused by the fact that, despite this higher void volume, ZIF-7 is significantly less accessible for $\mathrm{CO}_{2}$ than ZIF-8 and ZIF-90, due to its
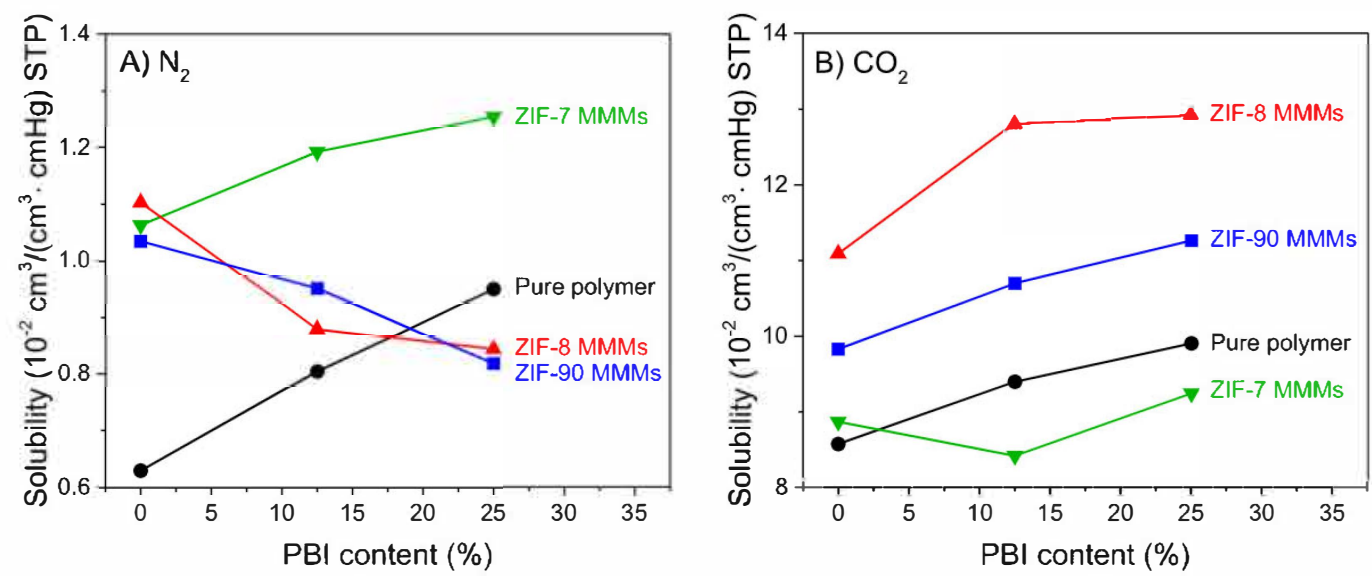

Fig. 6. A) $\mathrm{N}_{2}$ and B) $\mathrm{CO}_{2}$ solubility as function of the membrane PBI content in pure polymer membranes and the ZIF MMMs measured at 5 bar and $35{ }^{\circ} \mathrm{C}$. 
narrow pore aperture of $2.9 \AA$, resulting in ZIF-7 MMM $\mathrm{CO}_{2}$ solubilities that are in the range of the pure polymer membranes. The trends of the $\mathrm{CO}_{2}$ uptake in the MMMs thus highlight that the size of the ZIF pore aperture and volume and the ZIF linker flexibility are critical parameters that determine the $\mathrm{MMM} \mathrm{CO}_{2}$ solubility. Similar behavior regarding the size of the ZIF pore aperture and volume was observed as well for another isoreticular ZIF series in Matrimid [67].

\subsubsection{Membrane $\mathrm{CO}_{2} / \mathrm{N}_{2}$ separation factor and $\mathrm{CO}_{2}$ permeability}

Analysis of the mixed gas $\mathrm{CO}_{2} / \mathrm{N}_{2}$ separation performance of the pure polymer membranes and the MMMs clearly shows the effect of PBI addition to the membranes (Fig. 7).

3.3.2.1. Effect of $P B I$ incorporation on the $\mathrm{CO}_{2} / \mathrm{N}_{2}$ separation factor. The ZIF-7 and ZIF-8 MMMs clearly show that increasing the PBI content from $0 \%$ to $25 \%$ results in an increased $\mathrm{CO}_{2} / \mathrm{N}_{2}$ separation factor of $15 \%$ and $39 \%$, respectively. Since improved MOF-matrix interfaces improve the MMM separation factor it is concluded that PBI effectively compatibilizes the MOF-matrix interface in the ZIF-7 and ZIF-8, which is in agreement with the behavior of the glass transition temperatures of these MMMs (Table 3). To specify, it ishypothesized that this behavior is caused by the favorable interactions between the inidazole functionalities of these ZIFs and the PBI (as shown by Yang et al. for ZIF-7 and mPBI [20]) where the increased selectivity is typical for highly compatible interfaces [68]. Since these interactions are not present in the pure Matrimid MMMs, where only non-covalent interactions between the additives and the matrix are present [62], the compatibilizing effect is not observed for these MMMs. Similar effects of interfacial compatiblization were observed as well in other ZIF-8-based MMMs, where improved ZIF-matrix interactions increased the selectivity/separation factor $[14,15,69,70]$. On the other hand, the separation factor of the pure polymer membranes and the ZIF-90 MMMs is not affected by PBI incorporation, relative to the membranes without PBI addition. The separation factor of the ZIF-90 MMM without PBI relative to the pure polymer Matrimid membrane shows that, just as for the ZIF-7 and ZIF-8 MMMs without PBI, the MOF-matrix interface contains defects that lower the membrane selectivity [68]. However, in contrast with the ZIF-7 and ZIF-8 MMMs, PBI incorporation in the ZIF-90 MMMs does not enhance the membrane separation factor. This means that PBI does not improve the MOF-natrix interface in the ZIF-90 MMMs and complies

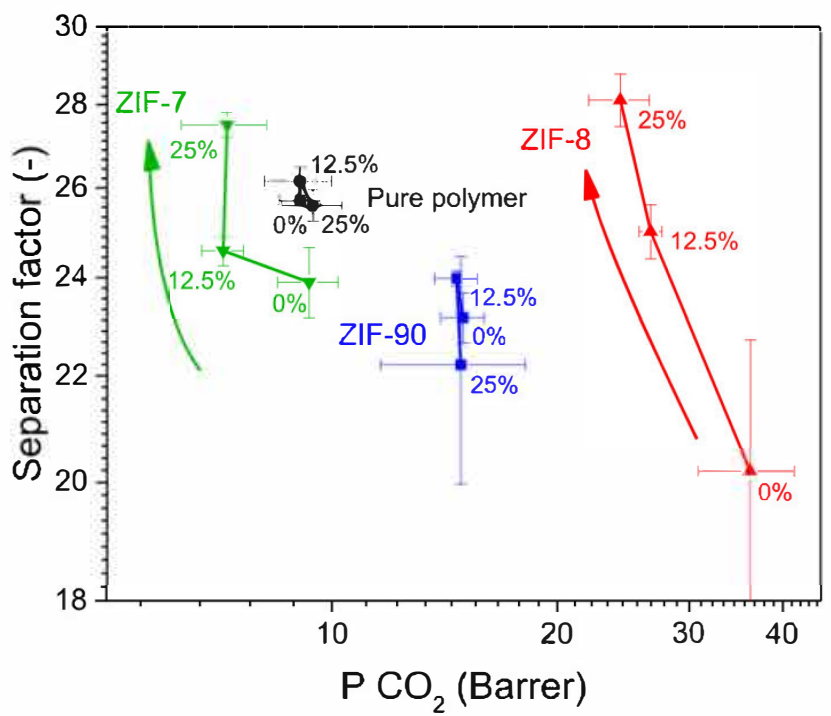

Fig. 7. Mixed gas $\mathrm{CO}_{2} / \mathrm{N}_{2}$ separation perfonance of the pure polymer membranes and the ZIF MMMs, measured at $35^{\circ} \mathrm{C}$ and 10 bar feed pressure with a 50:50 $\mathrm{CO}_{2} / \mathrm{N}_{2}$ composition. The percentages indicate the PBI content in the membranes. The scale of both axis is logarithmic. with the trend of the ZIF-90 glass transition temperatures and is attributed to the carboxaldehyde functionality of the ZIF-90 linker. As this functionality distorts the electron delocalization of the Ica linker and increases the acidity of the Ica proton, interactions with the matrix via $\pi-\pi$ stacking and imidazole activation of the PBI in the matrix are linited, respectively, resulting in interfacial incompatibilities and consequently a decreased selectivity [68], relative to the pure polymer membranes.

3.3.2.2. Effect of $P B I$ incorporation on the $\mathrm{CO}_{2}$ permeability. Incorporating PBI in the membranes affects the ZIF-7 and ZIF-8 $\mathrm{MMM} \mathrm{CO}_{2}$ permeability, but does not influence the pure polymer membrane and the ZIF-90 MMM CO 2 permeability. To specify, the ZIF-7 and ZIF-8 MMM CO 2 permeability decreases as function of the PBI content in the membrane (22 \% and $33 \%$ for the ZIF-7 and ZIF-8 MMMs from 0 to $25 \%$ PBI, respectively), while the $\mathrm{CO}_{2}$ permeability of the pure polymer membranes and ZIF-90 MMM is approximately constant as function of the PBI content. Just as for the MMM separation factors, these different effects of $\mathrm{PBI}$ addition to the MMMs on the $\mathrm{CO}_{2}$ permeability show that PBI compatibilizes the ZIF-7 and ZIF-8-matrix interface but does not improve the ZIF-90-matrix interface, where the previously mentioned molecular interactions in the MMMs are accounted for this behavior as well, since high compatibilities and interfacial defects result in a decreased and increased membrane permeability, respectively [68]. For the pure polymer membranes, the insensitivity of the $\mathrm{CO}_{2}$ permeability on the PBI content is as expected. Since it was shown by Kumbharkar et al. that increasing the chain mobility in a series of systematically modified PBI lowered the glass transition temperatures and consequently increased the permeability of the membranes (within the range of two orders of magnitude) [31], this behavior aligns with the $\mathrm{T}_{g}$ and $\mathrm{CO}_{2}$ permeability observed in this work, relative to conventional $\mathrm{m}$-PBI. Otherwise specified, the biphenylether moiety in the PBI used in this work will have a $\mathrm{CO}_{2}$ permeability within the range of Matrimid.

3.3.2.3. MMM separation performance relative to literature. Summarizing, these different effects PBI addition on the MMM separation factor and permeability show that only ZIF-7 and ZIF-8 with non-polar linker functionalities (Fig. 1) interact favorably with PBI, while this is not the case ZIF-90 with polar aldehyde groups (Fig. 1). In perspective, the extent of the effect of ZIF-matrix interfacial compatibilization on the MMM separation performance depends on the strategy of ZIF-matrix interfacial compatibilization. Shahid et al. improved the ZIF-8 MMM separation factor and permeability by grafting imidazole moieties on the polymer matrix that linked with ZIF-8 relative to the non-grafted ZIF-8 MMMs [14,15]. Yu et al. covalently bonded ZIF-8 to the matrix by introducing amine functionalities that bonded with carbonyl chloride moieties in the matrix, which increased the selectivity and permeability relative to non-functionalized ZIF-8 MMMs [69]. Li et al. compatibilized the ZIF-8-matrix interface by distributing an imidazole-based ionic liquid in ZIF-8 prior to the MMM fabrication, which increased the selectivity but decreased permeability relative to the non-compatibilized MMMs [70]. These studies in combination with this work show an interesting trend regarding the type of ZIF-matrix compatibilization, i.e., covalent approaches increase both the membrane selectivity and permeability, while dispersion approaches increase the membrane selectivity but decrease the permeability.

3.3.2.4. Influence of the ZIF pore dimensions on the separation performance. Besides the influence of PBI addition to the membranes, Fig. 7 illustrates the influence of the ZIF pore dimensions on the MMM permeability. The incorporation of both ZIF-8 and ZIF-90 in the MMMs significantly enhances the $\mathrm{CO}_{2}$ permeability relative to the pure polymer membranes as the size of the ZIF-8 and ZIF-90 pore apertures (Fig. 1), which is bigger than the kinetic diameter of $\mathrm{CO}_{2}(3.3 \AA)$, facilitates highly permeable paths [71]. Additionally, the significant difference in 
$\mathrm{CO}_{2}$ permeability between the ZIF-8 and ZIF-90 MMMs is attributed to the high rotatability of the ZIF-8 linker relative to the ZIF-90 linkers that both facilitates both an increased gas uptake and diffusivity $[55,56]$. Contrary to the increased ZIF-8 and ZIF-90 MMM CO 2 permeabilities, the ZIF-7 MMMs have a $\mathrm{CO}_{2}$ perneability equal to or lower than the Matrinid membranes, depending on the PBI content. This ineffectiveness of ZIF-7 incorporation on the $\mathrm{MMM} \mathrm{CO}_{2}$ permeability is caused by the size of the ZIF-7 pore aperture (Fig. 1), which is smaller than the kinetic diameter of $\mathrm{CO}_{2}$, impeding permeation through ZIF-7. These relationships between the size of the ZIF pore apertures and the MMM permeabilities aligns with literature where ZIF/Matrimid MMMs containing an isoreticular gnelinite ZIF series exhibited a similar relationship [67].

\subsubsection{Membrane $\mathrm{N}_{2}$ and $\mathrm{CO}_{2}$ diffusivities}

To gain more insight in the origin of the membrane separation performances of the different MMMs, the membrane diffusivities are provided and discussed in the following section. Fig. 8 shows the $\mathrm{N}_{2}$ and $\mathrm{CO}_{2}$ diffusivities of the pure polymer membranes and the ZIF MMMs with different PBI contents. The data clearly highlights the effect of ZIF dimensions. ZIF-8 and ZIF-90 with pore apertures bigger than the $\mathrm{CO}_{2}$ kinetic diameter (3.3 $\AA$ [71]) enhance the $\mathrm{CO}_{2}$ diffusivity, while ZIF-7 with a pore aperture smaller than the $\mathrm{CO}_{2}$ kinetic diameter decreases the diffusivity. Intuitively, the membrane $\mathrm{N}_{2}$ diffusivity should not be enhanced by the incorporation of ZIF-8 or ZIF-90 as the kinetic diameter of $\mathrm{N}_{2}$ (3.64 $\AA$ [71]) is significantly larger than the pore apertures of these ZIFs. However, both ZIFs are able to enhance the $\mathrm{N}_{2}$ diffusivity due to the linker rotatability of both ZIFs [55,56]. Additionally, as the linker rotatability of ZIF-8 is significantly higher than the ZIF-90 linker rotatability, the incorporation of ZIF-8 enhances the $\mathrm{N}_{2}$ and $\mathrm{CO}_{2}$ diffusivity more than ZIF-90, relative to the pure polymer membranes $[55,56]$. Clearly, the size of the ZIF pore aperture relative to the gas kinetic diameter and the linker rotatability is a guiding parameter that determines how the MMM diffusivity is affected by the ZIF incorporation. The difference in order of magnitude between the $\mathrm{N}_{2}$ and $\mathrm{CO}_{2}$ diffusivity in all membranes is attributed to the different kinetic diameters of the respective gases, where the smaller $\mathrm{CO}_{2}$ experiences less resistance migrating through the membranes than $\mathrm{N}_{2}$.

\subsubsection{Relative membrane $\mathrm{N}_{2}$ and $\mathrm{CO}_{2}$ diffusivities}

To analyze the effect of the PBI incorporation in the MMMs the $\mathrm{N}_{2}$ and $\mathrm{CO}_{2}$ diffusivity of the pure polymer membranes and the MMMs as function of the PBI content is presented in Fig. 9. The relative change in gas diffusivity highlights the effect of the incorporation of PBI in the polymer matrix. A higher amount of PBI decreases the relative $\mathrm{N}_{2}$ diffusivity of the pure polymer membranes, the ZIF-7 MMMs and the ZIF-8 MMMs while it increases the relative ZIF-90 MMM $\mathrm{N}_{2}$ diffusivity.
Since incompatibility between the MOF-matrix interface especially increases the diffusivity of slow gases relative to their bulk diffusivity, this means that the increase in the ZIF-90 MMM N $\mathrm{N}_{2}$ diffusivity as function of PBI content is caused by an increased ZIF-90-matrix incompatibility upon PBI addition [72]. This conclusion aligns with the significant decrease in $\mathrm{T}_{\mathrm{g}}$ of the ZIF-90 MMMs (Table 3 ) and substantiates that PBI addition to the ZIF-90 MMMs does not compatibilize the ZIF-90-matrix interface. Inversely, the fact that the ZIF-7 and ZIF-8 MMM $\mathrm{N}_{2}$ diffusivities decrease with increasing PBI content, shows that PBI improves the ZIF-matrix interactions in these MMMs [72].

Contrary to the relative $\mathrm{N}_{2}$ diffusivities, the incorporation of PBI decreases the $\mathrm{CO}_{2}$ diffusivity of all membranes. Moreover, this decrease is strongest for the ZIF-7 and ZIF-8 MMMs, while it is less and very comparable for both the pure polymer membranes and the ZIF-90 MMMs. This insensibility of the ZIF-90 MMM $\mathrm{CO}_{2}$ diffusivity to the PBI content relative to the pure polymer membranes means that PBI only affects the $\mathrm{CO}_{2}$ diffusivity through the bulk polymer matrix and not that through the ZIF-90-matrix interface. Combined with the increased relative change in relative $\mathrm{N}_{2}$ diffusivity of the ZIF-90 MMMs, this observation substantiates that the ZIF-90-Matrinid compatibility is very low and addition of PBI addition does not improve that. Oppositely, the stronger relative decrease in the ZIF-7 and ZIF-8 MMM CO 2 diffusivities compared to that of the pure polymer membranes confirms that in these cases PBI addition improves the MOF-matrix interactions. Interestingly, combining the ZIF-7 and ZIF-8 MMM solubilities, separation performances and relative diffusivities reveals that the increased separation factors upon PBI incorporation are caused by different compatibilization mechanisms. While it is evident that for both MMMs the permeability is reduced upon PBI incorporation because of the significant reduction in diffusivities (Figs. 8 and 9), this is not the case for the separation factors (Fig. 7). For the ZIF-7 MMM the increased separation factor upon PBI incorporation stems from the fact that the $\mathrm{CO}_{2}$ diffusivity is reduced less than the $\mathrm{N}_{2}$ diffusivity, while for the ZIF- 8 MMM the increased separation factor upon PBI incorporation stems from the fact that the $\mathrm{N}_{2}$ solubility is decreased while the $\mathrm{CO}_{2}$ solubility is increased (Fig. 6). Since ZIF-8 has a bigger pore aperture than ZIF-7 (3.4 and $2.9 \AA$ A, respectively), it is hypothesized that PBI either intrudes the ZIF-8 pores lowering the diffusivity of $\mathrm{CO}_{2}$ into ZIF-8 or limits the linker rotatability at the ZIF-8 surface that both facilitate an increased sorption selectivity of the ZIF-8 particles in the matrix as this intrusion or linited linker rotatability both limit the uptake of $\mathrm{N}_{2}$ in ZIF-8.

The different effects of PBI addition to the MMMs on the membrane sorption, separation performance and the diffusivity in combination with the previously mentioned literature underscore that tailoring the MMM interface is an essential aspect for the design of MMMs with improved separation perfornances. In addition to this statement, the authors want to address that the development of methods to probe the
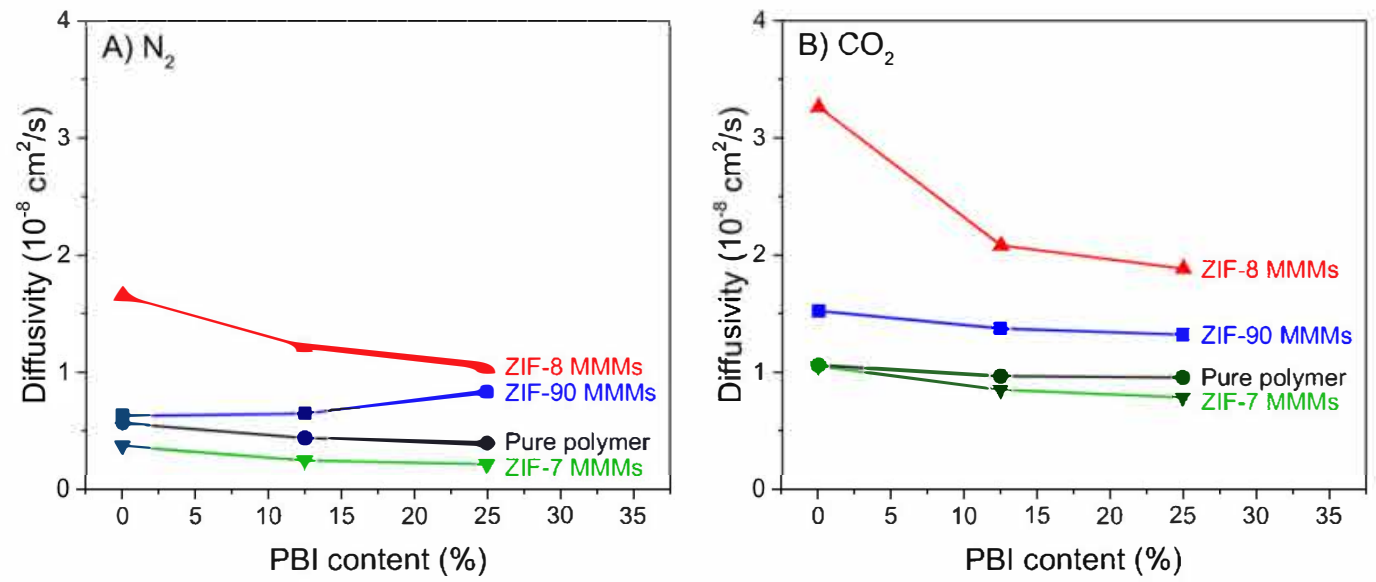

Fig. 8. Diffusivity of A) $\mathrm{N}_{2}$ and B) $\mathrm{CO}_{2}$ of the pure polymer membranes and the ZIF MMMs as function of the menbrane PBI content. 

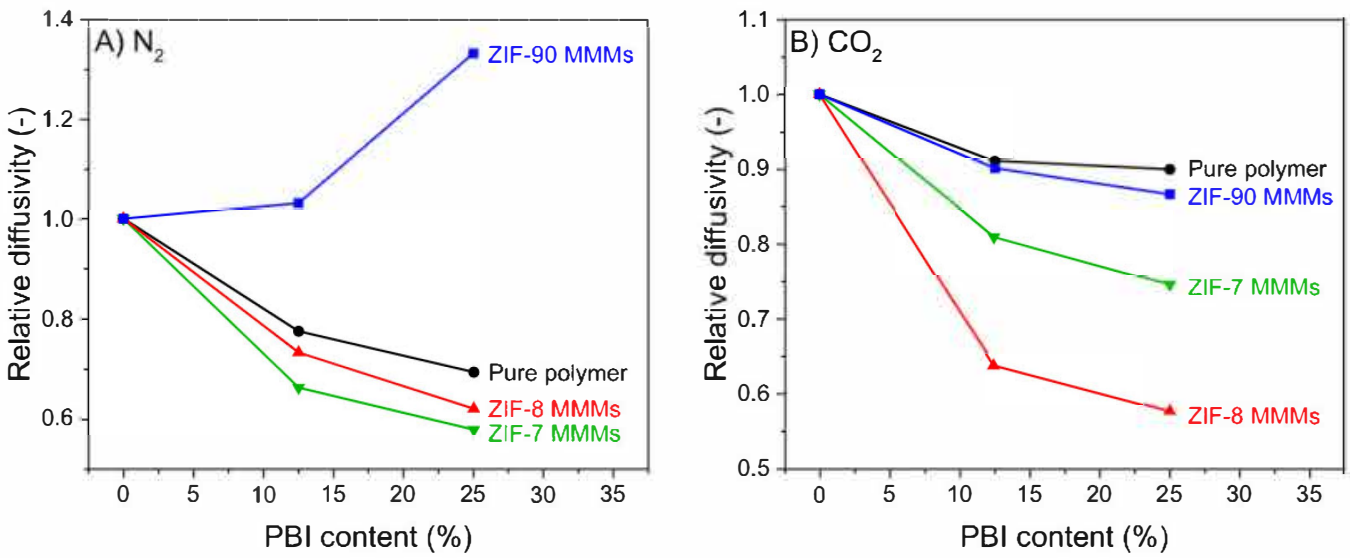

Fig. 9. Relative change in diffusivity of A) $\mathrm{N}_{2}$ and B) $\mathrm{CO}_{2}$ of the pure polymer membranes and the ZIF MMMs as function of the menbrane PBI content.

interfacial phenomena is essential for further development of MOFbased MMMs, since the interfacial structure-property relationships are nowadays based on relating the MMM separation perfornance directly to the MMM system, underexposing the influence of nicroscopic phenomena.

\section{Conclusion}

In this work the influence of PBI addition, a polymer with structural similarity to ZIFs, on ZIF-polymer matrix compatibility in MMMs was investigated. Three isoreticular ZIFs, with varying pore dinensions and functionality were used as microporous additives in Matrinid based matrices with varying PBI content. From these ZIFs, ZIF-8 exhibited the highest gas uptake for $\mathrm{N}_{2}$ and $\mathrm{CO}_{2}$ followed by ZIF-90 and ZIF-7. This trend follows the pore geometries and linker flexibility of the ZIFs and was reflected as well in the ZIF MMM $\mathrm{CO}_{2}$ permeabilities. The effect of PBI incorporation in the ZIF/Matrinid MMMs was dependent on the nature of the different ZIFs, i.e., the ZIF-7 and ZIF-8 MMMs showed an increase in $\mathrm{CO}_{2} / \mathrm{N}_{2}$ selectivity and a declining $\mathrm{CO}_{2}$ perneability with increasing PBI content, while the ZIF-90 MMM perneation behavior was rather uninfluenced by PBI addition. The increased $\mathrm{CO}_{2} / \mathrm{N}_{2}$ separation factor and decreased $\mathrm{CO}_{2}$ permeability with increasing PBI content in the ZIF-7 and ZIF-8 MMMs was caused by the compatibilizing effect of PBI at the ZIF-matrix interface. This effect was especially of significance on the highly perneable ZIF-8 MMMs as the $\mathrm{CO}_{2}$ diffusivity changed drastically upon PBI incorporation. These observations resulted in the following two conclusions: (1) the potential of PBI addition on the ZIF based MMM separation performance is dependent on the nature of the ZIF and (2) when PBI addition influences the ZIF based MMM permeation behavior, this is due to a change in the MOF-matrix interface. These conclusions show that tuning the MMM perfornance towards applications is a delicate process, where the influence of the compatibility between the MOF and the matrix is a critical design aspect that plays a significant role in the final membrane performance.

\section{Author statement}

The authors declare that all authors have seen and approved the final version of the submitted manuscript.

\section{CRediT authorship contribution statement}

Machiel van Essen: Conceptualization, Methodology, Validation, Fornal analysis, Investigation, Writing - original draft, Writing - review \& editing, Visualization. Raymond Thür: Fornal analysis, Investigation, Writing - review \& editing. Luuk van den Akker: Fornal analysis, Investigation. Menno Houben: Formal analysis, Investigation, Writing - review \& editing. Ivo F.J. Vankelecom: Writing - review \& editing.
Kitty Nijmeijer: Conceptualization, Supervision, Writing - review \& editing, Project administration, Funding acquisition. Zandrie Borneman: Conceptualization, Supervision, Writing - review \& editing.

\section{Declaration of competing interest}

The authors declare that they have no known competing financial interests or personal relationships that could have appeared to influence the work reported in this paper.

\section{Acknowledgements}

This work is part of the research program 'Fuel feedstock production by a combined approach of controlled plasma conversion and membrane separation' with project number 13584, which is partly financed by the Dutch Research Council (NWO). R.T. acknowledges FWO Vlaanderen for a SB PhD grant $(1 \mathrm{~S} 63317 \mathrm{~N})$.

\section{Appendix A. Supplementary data}

Supplementary data to this article can be found online at https://doi. org/10.1016/j.mensci.2021.119642.

\section{References}

[1] H.B. Tanh Jeazet, C. Staudt, C. Janiak, Metal-ørganic frameworks in mixed-matrix membranes for gas separation, Dalton Trans. 41 (2012) 14003-14027, https://doi. org/10.1039/c2dt31550e.

[2] M. Vinoba, M. Bhagiyalakshmi, Y. Alqaheem, A.A. Alomair, A. Pérez, M.S. Rana, Recent progress of fillers in mixed matrix membranes for CO 2 separation : a review, Separ. Purif. Technol. 188 (2017) 431-450, https://doi.org/10.1016/j. seppur.2017.07.051.

[3] S.R. Venna, M.A. Carreon, Metal organic framework membranes for carbon dioxide separation, Chem. Eng. Sci. 124 (2015) 3-19, https://doi.org/10.1016/j. ces.2014.10.007.

[4] B.R. Pimentel, A. Parulkar, E. Zhou, N.A. Brunelli, R.P. Lively, Zeolitic imidazolate frameworks: next-generation materials for energy-efficient gas separations, ChemSusChem 7 (2014) 3202-3240, https://doi.org/10.1002/cssc.201402647.

[5] W. Guan, Y. Dai, X. Yang, Y. Xi, Zeolite imidazolate framework (ZIF)-based mixed matrix membranes for CO2 separation: a review, J. Appl. Polym. Sci. (2020) 1-13, https://doi.org/10.1002/app.48968, 48968.

[6] B. Chen, Z. Yang, Y. Zhu, Y. Xia, Zeolitic imidazolate framework materials: recent progress in synthesis and applications, J. Mater. Chem. A. 2 (2014) 16811-16831, https://doi.org/10.1039/C4TA02984D.

[7] K.S. Park, Z. Ni, A.P. Côté, J.Y. Choi, R. Huang, F.J. Uribe-Romo, H.K. Chae, M. O'Keeffe, O.M. Yaghi, Exceptional chemical and thermal stability of zeolitic imidazolate frameworks, Proc. Natl. Acad. Sci. U.S.A. 103 (2006) 10186-10191, https://doi.org/10.1073/pnas.0602439103.

[8] W. Morris, C.J. Doonan, H. Furukawa, R. Banerjee, O.M. Yaghi, Crystals as molecules: postsynthesis covalent functionalization of zeolitic imidazolate frameworks, J. Am. Chem. Soc. 130 (2008) 12626-12627, https://doi.org/ 10.1021/ja805222x.

[9] Q. Qian, P.A. Asinger, M.J. Lee, G. Han, K.M. Rodriguez, S. Lin, F.M. Benedetti, A. X. Wu, W.S. Chi, Z.P. Smith, MOF-based membranes for gas separations, Chem. Rev. 120 (2020) 8161-8266, https://doi.org/10.1021/acs.chemrev.0c00119. 
[10] A.U. Ortiz, A.P. Freitas, A. Boutin, A.H. Fuchs, F.-X. Coudert, What makes zeolitic imidazolate frameworks hydrophobic or hydrophilic? The impact of geometry and functionalization on water adsorption, Phys. Chem. Chem. Phys. 16 (2014) 9940-9949, https://doi.org/10.1039/c3cp54292k.

[11] J. Yao, H. Wang, Zeolitic imidazolate framework composite membranes and thin films: synthesis and applications, Chem. Soc. Rev. 43 (2014) 4470-4493, https:// doi.org/10.1039/c3es60480b.

[12] R. Castro-muñoz, V. Fíla, V. Martin-gil, C. Muller, Enhanced CO2 permeability in Matrimid $\$ 5218$ mixed matrix membranes for separating binary $\mathrm{CO} 2 / \mathrm{CH} 4$ mixtures, Separ. Purif. Technol. 210 (2019) 553-562, https://doi.org/10.1016/j. seppur.2018.08.046.

[13] S. Basu, A. Cano-Odena, I.F.J. Vankelecom, MOF-containing mixed-matrix membranes for $\mathrm{CO} 2 / \mathrm{CH} 4$ and $\mathrm{CO} 2 / \mathrm{N} 2$ binary gas mixture separations, Separ. Purif. Technol. 81 (2011) 31-40, https://doi.org/10.1016/j.seppur.2011.06.037.

14] S. Shahid, K. Nijmeijer, S. Nehache, I. Vankelecom, A. Deratani, D. Quemener, MOF-mixed matrix membranes: precise dispersion of MOF particles with better compatibility via a particle fusion approach for enhanced gas separation properties, J. Membr. Sci. 492 (2015) 21-31, https://doi.org/10.1016/j. memsci.2015.05.015.

[15] S. Shahid, K. Nijmeijer, Performance and plasticization behavior of polymer-MOF membranes for gas separation at elevated pressures, J. Membr. Sci. 470 (2014) 166-177, https://doi.org/10.1016/j.memsci.2014.07.034.

[16] M.J.C. Ordoñez, K.J. Balkus, J.P. Ferraris, I.H. Musselman, Molecular sieving realized with ZIF-8/Matrimid@ mixed-matrix membranes, J. Membr. Sci. 361 (2010) 28-37, https://doi.org/10.1016/j.memsci.2010.06.017.

[17] Q. Song, S.K. Nataraj, M.V. Roussenova, J.C. Tan, D.J. Hughes, W. Li, P. Bourgoin, M.A. Alam, A.K. Cheetham, S.A. Al-Muhtaseb, E. Sivaniah, Zeolitic imidazolate framework (ZIF-8) based polymer nanocomposite membranes før gas separation, Energy Environ. Sci. 5 (2012) 8359-8369, https://doi.org/10.1039/c2ee21996d.

[18] T. Yang, T. Chung, High performance ZIF-8/PBI nano-composite membranes for high temperature hydrogen separation consisting of carbon monoxide and water vapor, Int. J. Hydrogen Energy 38 (2012) 229-239, https://doi.org/10.1016/j. ijhydene.2012.10.045.

19] J. Sánchez-Laínez, B. Zornoza, S. Friebe, J. Caro, S. Cao, A. Sabetghadam, B. Seoane, J. Gascon, F. Kapteijn, C. Le Guillouzer, G. Clet, M. Daturi, C. Téllez, J. Coronas, Influence of ZIF-8 particle size in the performance of polybenzimidazole mixed matrix membranes for pre-combustion $\mathrm{CO} 2$ capture and its validation through interlaboratory test, J. Membr. Sci. 515 (2016) 45-53, https://doi.org/10.1016/j.memsci.2016.05.039.

[20] T. Yang, Y. Xiao, T. Chung, Poly-/metal-benzimidazole nano-composite membranes for hydrogen purification, Energy Environ. Sci. 4 (2011) 4171-4180, https://doi.org/10.1039/c1ee01324f

[21] T. Yang, T. Chung, Room-temperature synthesis of ZIF-90 nanocrystals and the derived nano-composite membranes for hydrogen separation, J. Mater. Chem. A. 1 (2013) 6081-6090, https://doi.org/10.1039/c3ta10928c

[22] T. Yang, G.M. Shi, T.S.C. Chung, Symmetric and asymmetric zeolitic imidazolate frameworks (ZIFs)/Polybenzimidazole (PBI) nanocomposite membranes for hydrogen purifi cation at high temperatures, Adv. Energy Mater. 2 (2012) 1358-1367, https://doi.org/10.1002/aenm.201200200.

[23] S.C. Kumbharkar, P.B. Karadkar, U.K. Kharul, Enhancement of Gas Permeation Properties of Polybenzimidazoles by Systematic Structure Architecture, vol. 286, 2006, pp. 161-169, https://doi.org/10.1016/j.memsci.2006.09.030.

24] R. Lin, B. Villacorta Hernandez, L. Ge, Z. Zhu, Metal organic framework based mixed matrix membranes: an overview on filler polymer interfaces, J. Mater. Chem. A. 6 (2018) 293-312, https://doi.org/10.1039/c7ta07294e.

[25] L. Xiang, L. Sheng, C. Wang, L. Zhang, Y. Pan, Y. Li, Amino-functionalized ZIF-7 nanocrystals: improved intrinsic separation ability and interfacial compatibility in mixed-matrix membranes for $\mathrm{CO} 2 / \mathrm{CH} 4$ separation, Adv. Mater. 29 (2017) 1-8, https://doi.org/10.1002/adma.201606999.

[26] M. Balçık, S.B. Tantekin-ersolmaz, M.G. Ahunbay, Interfacial analysis of mixedmatrix membranes under exposure to high-pressure CO2, J. Membr. Sci. 607 (2020) 1-10, https://doi.org/10.1016/j.memsci.2020.118147.

[27] A. Knebel, A. Bavykina, S.J. Datta, L. Sundermann, L. Garzon-tovar, Y. Lebedev, S. Durini, R. Ahmad, S.M. Kozlov, G. Shterk, M. Karunakaran, I.D. Carja, D. Simic, I. Weilert, M. Klüppel, U. Giese, L. Cavallo, M. Rueping, M. Eddaoudi, J. Caro, J. Gascon, Solution processable metal-organic frameworks for mixed matrix membranes using porous liquids, Nat. Mater. 19 (2020) 1346-1353, https://doi. org/10.1038/s41563-020-0764-y.

[28] L. Dong, M. Chen, J. Li, D. Shi, W. Dong, X. Li, Y. Bai, Metal-organic frameworkgraphene oxide composites : a facile method to highly improve the CO 2 separation performance of mixed matrix membranes, J. Membr. Sci. 520 (2016) 801-811, https://doi.org/10.1016/j.memsci.2016.08.043.

[29] A. Ozcan, R. Semino, G. Maurin, A.O. Yazaydin, Modeling of gas transport through polymer/MOF Interfaces : a microsecond-scale concentration gradient-driven molecular dynamics study, Chem. Mater. 32 (2020) 1288-1296, https://doi.org/ 10.1021/acs.chemmater.9b04907.

[30] J. Büsselmann, M. Rastedt, T. Klicpera, K. Reinwald, H. Schmies, A. Dyck, P. Wagner, Analysis of HT-PEM MEAs' long-term stabilities, Energies 13 (2020) $1-16$.

[31] S.C. Kumbharkar, U.K. Kharul, Investigation of gas permeation properties of systematically modified polybenzimidazoles by N-substitution, J. Membr. Sci. 357 (2010) 134-142, https://doi.org/10.1016/j.memsci.2010.04.014.

[32] S. Shahid, K. Nijmeijer, Matrimid /polysulfone blend mixed matrix membranes containing ZIF-8 nanoparticles for high pressure stability in natural gas separation, Separ. Purif. Technol. 189 (2017) 90-100, https://doi.org/10.1016/j. seppur.2017.07.075
[33] R. Thür, N. Van Velthoven, V. Lemmens, M. Bastin, S. Smolders, D. De Vos, I.F. J. Vankelecom, Modulator-mediated functionalization of MOF-808 as a platform tool to create high-performance mixed-matrix membranes, ACS Appl. Mater. Interfaces 11 (2019) 44792-44801, https://doi.org/10.1021/acsami.9b19774.

[34] J. Didden, R. Thür, A. Volodin, I.F.J. Vankelecom, Blending PPO-based molecules with Pebax MH 1657 in membranes for gas separation, J. Appl. Polym. Sci. 135 (2018) 1-12, https://doi.org/10.1002/app.46433.

[35] G. Genduso, B.S. Ghanem, I. Pinnau, Experimental mixed-gas permeability , sorption and diffusion of $\mathrm{CO} 2-\mathrm{CH} 4$ mixtures in 6FDA-mPDA polyimide membrane: unveiling the effect of competitive sorption on permeability selectivity, Membranes 9 (2019) 1-14, https://doi.org/10.3390/membranes9010010.

[36] A.E. Gemeda, M. Grazia De Angelis, N. Du, N. Li, M.D. Guiver, G.C. Sarti, Mixed gas sorption in glassy polymeric membranes. III. $\mathrm{CO} 2 / \mathrm{CH} 4$ mixtures in a polymer of intrinsic microporosity (PIM-1): effect of temperature, J. Membr. Sci. 524 (2017) 746-757, https://doi.org/10.1016/j.memsci.2016.11.053.

[37] A. Schejn, L. Balan, V. Falk, L. Aranda, G. Medjahdi, R. Schneider, Controlling ZIF8 nano- and microcrystal formation and reactivity through zinc salt variations, CrystEngComm 16 (2014) 4493-4500, https://doi.org/10.1039/c3ce42485e.

[38] N.A.H.M. Nordin, A.F. Ismail, A. Mustafa, P.S. Goh, D. Rana, T. Matsuura, Aqueous room temperature synthesis of zeolitic imidazole framework 8 (ZIF-8) with various concentrations of triethylamine, RSC Adv. 4 (2014) 33292-33300, https://doi.org/ 10.1039/C4RA03593C.

[39] M. Thommes, K. Kaneko, A. V Neimark, J.P. Olivier, F. Rodriguez-reinoso, J. Rouquerol, K.S.W. Sing, Physisorption of gases, with special reference to the evaluation of surface area and pore size distribution (IUPAC Technical Report), Pure Appl. Chem. 87 (2015) 1051-1069, https://doi.org/10.1515/pac-2014-1117.

[40] T. Li, Y. Pan, K. Peinemann, Z. Lai, Carbon dioxide selective mixed matrix composite membrane containing ZIF-7 nano-fillers, J. Membr. Sci. 425-426 (2013) 235-242, https://doi.org/10.1016/j.memsci.2012.09.006.

[41] X. Wu, M. Niknam Shahrak, B. Yuan, S. Deng, Synthesis and characterization of zeolitic imidazolate framework ZIF-7 for $\mathrm{CO} 2$ and $\mathrm{CH} 4$ separation, Microporous Mesoporous Mater. 190 (2014) 189-196, https://doi.org/10.1016/j. micromeso.2014.02.016.

[42] F.K. Shieh, S.C. Wang, S.Y. Leo, K.C.W. Wu, Water-based synthesis of zeolitic imidazolate framework-90 (ZIF-90) with a controllable particle size, Chem. Eur J. 19 (2013) 11139-11142, https://doi.org/10.1002/chem.201301560.

[43] N.A.H.M. Nordin, A.F. Ismail, A. Mustafa, R. Surya Murali, T. Matsuura, The impact of ZIF-8 particle size and heat treatment on $\mathrm{CO} 2 / \mathrm{CH} 4$ separation using asymmetric mixed matrix membrane, RSC Adv. 4 (2014) 52530-52541, https:// doi.org/10.1039/c4ra08460h.

[44] C. Cuadrado-Collados, J. Fernández-Català, F. Fauth, Y.Q. Cheng, L.L. Daemen, A. J. Ramirez-Cuesta, J. Silvestre-Albero, Understanding the breathing phenomena in nano-ZIF-7 upon gas adsorption, J. Mater. Chem. A. 5 (2017) 20938-20946, https://doi.org/10.1039/c7ta05922a.

[45] S. Tanaka, K. Kida, M. Okita, Y. Ito, Y. Miyake, Size-Controlled synthesis of zeolitic imidazolate framework-8 (ZIF-8) crystals in an aqueous system at room temperature, Chem. Lett. 41 (2012) 1337-1339, https://doi.org/10.1246/ cl.2012.1337.

[46] Y. Zhang, Y. Jia, M. Li, L. Hou, Influence of the 2-methylimidazole/zinc nitrate hexahydrate molar ratio on the synthesis of zeolitic imidazolate framework-8 crystals at room temperature, Nat. Sci. Reports (2018) 1-7, https://doi.org/ 10.1038/s41598-018-28015-7

47] T.H. Bae, J.S. Lee, W. Qiu, W.J. Koros, C.W. Jones, S. Nair, A high-performance gas-separation membrane containing submicrometer-sized metal-organic framework crystals, Angew. Chem. Int. Ed. 49 (2010) 9863-9866, https://doi.org/ 10.1002/anie.201006141.

[48] S. Japip, Y. Xiao, T.-S. Chung, Particle-size effects on gas transport properties of 6FDA-durene/ZIF-71 mixed matrix membranes, Ind. Eng. Chem. Res. 55 (2016) 9507-9517, https://doi.org/10.1021/acs.iecr.6b02811.

[49] C. Graham, D.A. Imrie, R.E. Raab, Measurement of the electric quadrupole moments of CO2, CO, N2, Cl2 and BF3, Mol. Phys. 93 (1998) 49-56, https://doi. org/10.1080/002689798169429.

[50] C. Gücüyener, J. Van Den Bergh, J. Gascon, F. Kapteijn, Ethane/ethene separation turned on its head: selective ethane adsorption on the metal-organic framework ZIF-7 through a gate-opening mechanism, J. Am. Chem. Soc. 132 (2010) 17704-17706, https://doi.org/10.1021/ja1089765.

[51] M. Áhlén, A. Jaworski, M. Strømme, O. Cheung, Selective adsorption of CO2 and SF6 on mixed-linker ZIF-7-8s: the effect of linker substitution on uptake capacity and kinetics, Chem. Eng. J. 422 (2021) 1-15, https://doi.org/10.1016/j. cej.2021.130117, 130117.

[52] A. Arami-Niya, G. Birkett, Z. Zhu, T.E. Rufford, Gate opening effect of zeolitic imidazolate framework ZIF-7 for adsorption of $\mathrm{CH} 4$ and $\mathrm{CO} 2$ from N2, J. Mater. Chem. A. 5 (2017) 21389-21399, https://doi.org/10.1039/c7ta03755d.

[53] F.A. Beni, M.N. Shahrak, Alkali metals-promoted capacity of ZIF-8 and ZIF-90 for carbon capturing: a molecular simulation study, Polyhedron 178 (2020), 114338, https://doi.org/10.1016/j.poly.2019.114338.

[54] Y.-T. Liao, S. Dutta, C.-H. Chien, C.-C. Hu, F.-K. Shieh, C.-H. Lin, K.C.-W. Wu, Synthesis of mixed-ligand zeolitic imidazolate framework (ZIF-8-90) for $\mathrm{CO} 2$ adsorption, J. Inorg. Organomet. Polym. Mater. 25 (2015) 251-258, https://doi. org/10.1007/s10904-014-0131-z.

[55] D. Fairen-Jimenez, S.A. Moggach, M.T. Wharmby, P.A. Wright, S. Parsons, T. Duren, Opening the gate: framework flexibility in ZIF-8 explored by experiments and simulations, J. Am. Chem. Soc. 133 (2011) 8900-8902, https://doi.org/ $10.1021 / \mathrm{ja202154j}$. 
[56] B. Zheng, F. Fu, L.L. Wang, L. Yang, Y. Zhu, H. Du, Investigation of the Linker Swing Motion in the Zeolitic Imidazolate Framework ZIF-90, 2018, https://doi. org/10.1021/acs.jpcc.8b00018.

[57] K. Zhang, R.P. Lively, M.E. Dose, A.J. Brown, C. Zhang, J. Chung, S. Nair, J. Koros, R.R. Chance, Alcohol and water adsorption in zeolitic imidazolate frameworks, Chem. Commun. 49 (2013) 3245-3247, https://doi.org/10.1039/c3cc39116g.

[58] R. Castro-Muñoz, V. Martin-Gil, M.Z. Ahmad, V. Fíla, Matrimid 5218 in preparation of membranes for gas separation: current state-of-the-art, Chem. Eng. Commun. 205 (2018) 161-196, https://doi.org/10.1080/ 00986445.2017 .1378647$.

[59] S.S. Hosseini, M.M. Teoh, T.S. Chung, Hydrogen separation and purification in membranes of miscible polymer blends with interpenetration networks, Polymer 49 (2008) 1594-1603, https://doi.org/10.1016/j.polymer.2008.01.052.

[60] J. DeFelice, J.E.G. Lipson, The influence of additives on polymer matrix mobility and the glass transition, Soft Matter (2020) 46-49, https://doi.org/10.1039/ d0sm01634a.

[61] L. Diestel, N. Wang, B. Schwiedland, F. Steinbach, U. Giese, J. Caro, MOF based MMMs with enhanced selectivity due to hindered linker distortion, J. Membr. Sci. 492 (2015) 181-186, https://doi.org/10.1016/j.memsci.2015.04.069.

[62] E.M. Mahdi, J. Tan, Mixed-matrix membranes of zeolitic imidazolate framework (ZIF-8)/Matrimid nanocomposite: thermo-mechanical stability and viscoelasticity underpinning membrane separation performance, J. Membr. Sci. 498 (2016) 276-290, https://doi.org/10.1016/j.memsci.2015.09.066.

[63] J.A. Thompson, K.W. Chapman, W.J. Koros, C.W. Jones, S. Nair, Sonication induced Ostwald ripening of ZIF-8 nanoparticles and formation of ZIF-8/polymer composite membranes, Microporous Mesoporous Mater. 158 (2012) 292-299, https://doi. org/10.1016/j.micromeso.2012.03.052

[64] K. Ghosal, B.D. Freeman, Gas separation using polymer membranes: an overview, Polym. Adv. Technol. 5 (1994) 673-697, https://doi.org/10.1002/ pat.1994.220051102.
[65] A. Thran, G. Kroll, F. Faupel, Correlation between fractional free volume and diffusivity, J. Polym. Sci., Part B: Polym. Phys. 37 (1999) 3344-3358.

[66] S. Mazinani, R. Ramezani, G.F. Molelekwa, S. Darvishmanesh, R. Di, B. Van Der Bruggen, Plasticization suppression and CO 2 separation enhancement of Matrimid through homogeneous blending with a new high performance polymer, J. Membr. Sci. 574 (2019) 318-324, https://doi.org/10.1016/j.memsci.2018.12.060.

[67] M. van Essen, L. van den Akker, R. Thür, M. Houben, I.F.J. Vankelecom, Z. Borneman, K. Nijmeijer, The influence of pore aperture, volume and functionality of isoreticular gmelinite zeolitic imidazolate frameworks on the mixed gas $\mathrm{CO} 2 / \mathrm{N} 2$ and $\mathrm{CO} 2 / \mathrm{CH} 4$ separation performance in mixed matrix membranes, Separ. Purif. Technol. 260 (2021) 1-10, https://doi.org/10.1016/j. seppur.2020.118103.

[68] T.T. Moore, W.J. Koros, Non-ideal effects in organic-inorganic materials for gas separation membranes, J. Mol. Struct. 739 (2004) 87-98, https://doi.org/ 10.1016/j.molstruc.2004.05.043.

[69] S. Yu, S. Li, S. Huang, Z. Zeng, S. Cui, Y. Liu, Covalently bonded zeolitic imidazolate frameworks and polymers with enhanced compatibility in thin film nanocomposite membranes før gas separation, J. Membr. Sci. 540 (2017) 155-164, https://doi.org/10.1016/j.memsci.2017.06.047.

[70] H. Li, L. Tuo, K. Yang, H. Jeong, Y. Dai, G. He, W. Zhao, Simultaneous enhancement of mechanical properties and $\mathrm{CO} 2$ selectivity of ZIF-8 mixed matrix membranes : interfacial toughening effect of ionic liquid, J. Membr. Sci. 511 (2016) 130-142, https://doi.org/10.1016/j.memsci.2016.03.050.

[71] S. Matteucci, Y. Yampolskii, B.D. Freeman, I. Pinnau, Materials Science of Membranes for Gas and Vapor Separation Materials, John Wiley \& Sons, Ltd., West Sussex, 2006.

[72] R.C. Dutta, S.K. Bhatia, Interfacial engineering of MOF-based mixed matrix membrane through atomistic simulations, J. Phys. Chem. C 124 (2020) 594-604, https://doi.org/10.1021/acs.jpcc.9b09384. 